\title{
Traffic Equilibrium for Mixed Traffic Flows of Human-Driven Vehicles and Connected and Autonomous Vehicles in Transportation Networks under Tradable Credit Scheme
}

\author{
Fang Zhang, ${ }^{1,2,3}$ Jian $\mathrm{Lu}\left(\mathbb{D}^{\mathrm{D}}{ }^{1,2,3}\right.$ and Xiaojian $\mathrm{Hu}^{1,2,3}$ \\ ${ }^{1}$ Jiangsu Key Laboratory of Urban ITS, Southeast University, Nanjing, China \\ ${ }^{2}$ Jiangsu Province Collaborative Innovation Center of Modern Urban Traffic Technologies, Southeast University, Nanjing, China \\ ${ }^{3}$ School of Transportation, Southeast University, Nanjing 211189, China
}

Correspondence should be addressed to Jian Lu; lujian_1972@seu.edu.cn

Received 29 January 2020; Revised 10 May 2020; Accepted 18 May 2020; Published 2 June 2020

Academic Editor: Francesco Galante

Copyright (c) 2020 Fang Zhang et al. This is an open access article distributed under the Creative Commons Attribution License, which permits unrestricted use, distribution, and reproduction in any medium, provided the original work is properly cited.

In this paper, the traffic equilibriums for mixed traffic flows of human-driven vehicles (HDV) and connected and autonomous vehicles (CAV) under a tradable credit scheme (TCS) are established and formulated as two variational inequality (VI) problems with exogenous and endogenous CAV penetration rate, respectively. A modified Lagrangian dual (MLD) method embedded with a revised Smith's route-swapping (RSRS) algorithm is proposed to solve the problems. Based on the numerical analysis, the impacts of CAV penetration and the extra expense of using a CAV on network performance are investigated. A novel driveway management, autonomous vehicle/credit charge (AVCC) link, is put forward to improve the efficiency of TCS. Under the TCS with exogenous CAV penetration rate, a logit-based model is applied to describe the stochastic user equilibrium for mixed traffic flow. It is found that the penetration of CAV gives rise to a better network performance and it can be further improved by the deployment of AVCC link. Under the TCS with endogenous penetration rate, a nested-logit model is applied to describe travelers' choices of vehicle types and routes. It is found that the deployment of AVCC links can slow down the decline rate of CAV penetration with increasing expense and thus ensure a lower average travel time for CAVs. In both cases, the deployment of AVCC links can stimulate credit trading and drop down its unit price.

\section{Introduction}

Due to the potential benefits to transportation systems, autonomous vehicle (AV) technologies have gained tremendous amount of attention among researchers, industry leaders, and policy-makers in recent years. Since Google started its self-driving car project in 2009 [1], many Internet companies [2,3] and automakers [4] followed the suit to launch their own projects on self-driving. Correspondingly, countries including the United States, Australia, and China [5-7] have issued regulations related to road testing of AV to facilitate its development. All of these suggest that the ascending moment of AV technologies is coming.

Together with AVs, the construction of Cooperative Vehicle Infrastructure Systems (CVIS) is also a hot topic in the area of intelligent transportation. The vehicle-to-infrastructure and vehicle-to-vehicle communication technologies in CVIS make the vehicles connected and have a perfect knowledge on traffic conditions and other vehicles' information. More generally, connected and autonomous vehicles (CAV) refer to the vehicles with high level of connection and automation.

Studies have shown that CAVs have several advantages over human-driven vehicles (HDV). First, the penetration of CAVs can increase road capacity. Shladover et al. [8] conducted a series of simulations and found that CAV greatly increased capacity after its penetration reached moderate-tohigh percentages. Milanes et al. [9] showed that, in the context of wireless communication, the spacing gaps can be significantly reduced. Levin and Boyles [10] showed that even if a small proportion of HDVs are replaced by CAVs, the redistributed network flows can reduce average travel time significantly due to the enhanced capacity. Second, CAVs can enhance traffic safety. Ye and Yamamoto [11] demonstrated 
that CAVs could reduce the velocity difference between vehicles and greatly ease stop-and-go traffic and smooth the traffic flow. Papadoulis et al. [12] conducted a safety evaluation using the surrogate safety assessment model and found that the estimated traffic conflicts could be largely reduced by the penetration of CAVs. Third, CAVs can improve energy efficiency. Greenblatt and Saxena [13] pointed out that AVs could substantially reduce greenhouse gas emissions and petrol consumption even if total vehicle-miles traveled, average speed, and vehicle size increased. Mersky and Samaras [14] proposed a standardized method for testing the fuel economy effects of AV behavior when following another vehicle and found that automation could reduce fuel economy losses by $10 \%$. More detailed reviews on the CAV's benefits can be found in the works of Fagnant and Kockelman [15] and Zong [16].

Given the attractive advantages of CAV and government-supported developments of CVIS, it is expectable that CAVs will be publicly available and their penetration rate will keep growing rapidly since then. From researcher's standpoint, it is necessary to analyze the impacts of the CAV penetration into traffic flow with pure HDVs and study its interactions with HDVs and infrastructures. Only in doing so can we detect the potential problems and take corresponding measures beforehand.

\subsection{Literature Review}

1.1.1. CAV in Network Equilibrium Problem. Before the emergence of CAV and CVIS, the term advanced traveler information systems (ATIS) was proposed earlier before and had attracted considerable attention among researchers since then [17-23]. The ATIS is designed to help commuters compensate for their limited knowledge and thus make more reasonable travel choice decisions by providing traffic information for them. In light of this, travelers were divided into those equipped with ATIS and the unequipped. It was assumed that they had different perception variances on travel disutility due to the fact that they obtained different quality of traffic information. Based on this, many works published focused on the multiclass traffic assignment problems for equipped and unequipped vehicles and tried to find the equilibrium state of the mixed traffic flow.

From today's perspective, the vehicle equipped with ATIS can be basically regarded as a CAV with relatively low level of connection or automation. Hence the multiclass traffic assignment problems for equipped and unequipped vehicles can be recognized as a prototype of the multiclass traffic assignment for mixed HDVs and CAVs. Indeed, a large part of the assignment models proposed in recent years are further developed based on the previous ones, with different assumptions on the traveler's route choice behavior.

Given AV-dedicated lanes deployed in a general road network, Chen et al. [24] proposed a multiclass network equilibrium model, assuming the user equilibrium (UE) principles for route choices of both HDVs and CAVs. Combined with a diffusion model to forecast the evolution of endogenous CAV penetration over time, a time-dependent deployment model was further formulated to optimize the deployment plan of AV lanes. Furthermore, Chen et al. [25] extended AV lanes to AV zones, where only CAVs are allowed to use the links within the zone. Based on the assumption that CAVs followed different routing principles outside of and within the AV zones, a novel network equilibrium model and further a mixed-integer bilevel programming model were proposed to optimize the deployment of AV zones. Jiang [26] put forward a signal design problem for mixed equilibrium networks with HDVs and CAVs and formulated it as a bilevel program. The lowerlevel model describes a mixed equilibrium, where CAVs follow the Cournot-Nash principle and HDVs follow the UE principle. In the upper-level model, signal timings are optimized at signalized intersections to allocate appropriate green times to both HDVs and CAVs to minimize system travel cost. Zhang and Nie [27] proposed an optimal-ratio control scheme to improve transportation network efficiency by controlling the proportion of CAVs for each O-D pair. It was assumed that the HDVs and CAVs followed UE and system optimal principles, respectively. Wang et al. [28] proposed a multiclass traffic assignment model, where HDVs and CAVs follow different route choice principles, characterized by cross-nested-logit model and UE model, respectively. A route-swapping-based solution algorithm was developed to solve the problem efficiently.

Even though various models were adopted to characterize the travelers' route choice behavior in the context of mixed traffic, it is a consensus that there is a distinct gap for HDV and CAV between the information acquired for route choice. This is because a CAV is fully connected with infrastructure and other CAVs and has full knowledge of the network, making it more likely to choose the route with least cost compared with HDVs.

In this paper, we assume that travelers choose their routes according to stochastic user equilibrium (SUE) principle, and HDVs have a much larger perception variance on travel cost than CAVs. A logit model and a nested-logit model are proposed to describe the network equilibrium, which are developed based on Huang and Li's [29] and Wu etal.'s [30] works, respectively.

\subsubsection{Tradable Credit Scheme to Handle Transportation} Problems. A tradable credit scheme (TCS) was first applied in the realm of environmental policy, including the Kyoto Protocol and the European Union Emission Trading Scheme [31]. Since Yang and Wang [32] first developed quantitative analysis and modeling of TCS in a general network equilibrium context, an increasing number of research studies have been conducted to seek the potential benefits of TCS to mobility management [30, 33-39].

A TCS in the context of transportation aims to restrict the use of private cars in a specified area and time period. In the scheme, central authority distributes credits, representing a proportion of this total car use, to eligible travelers who need to redeem them when driving in the area. The scheme allows credits to be traded, activating a market mechanism that lets credits flow to those with the highest value of car use, whereas those with the lowest abatement costs will benefit by selling their credits. 
As an alternative to road pricing to handle transportation problems, the TCS is more advantaged in terms of public acceptability. On the one hand, a TCS is revenueneutral and the initial distribution of credits can be used to alleviate the unequal redistributive impacts, which can avoid the serious political resistance that would result if some group was favored over another. On the other hand, for travelers, they have a supplementary incentive to limit their vehicle use, because they can sell their extra credits as a compensation for the inconvenience.

More recently, many researchers have combined TCS with other traffic management methods or extended TCS to other kinds of applications. For example, Shirmohammadi and Yin [40] extended tradable credits to control the maximum queue length at a bottleneck. Miralinaghi and Peeta [41] proposed a multiperiod tradable credit scheme (TCS), in which travelers decide to consume or sell the credits in the current period or transfer to future periods. Bao et al. [42] proposed a novel kind of private financing of public road, build-equity-credit (BEC) scheme based on TCS, hoping to achieve a triple win for government, private firm, and travelers. Miralinaghi and Peeta [43] established a multiperiod TCS to minimize the vehicular emissions in a traffic network over a planning horizon. Later, with the same goal, the authors designed a robust multiperiod TCS to incentivize travelers to shift from internal combustion engine vehicles to zero-emissions vehicles [44]. Guo et al. [45] proposed a dynamic tradable credit scheme for control of the day-to-day evolution process of traffic flows in the network with elastic demand, where the distribution and charge of travel credits are adjusted from period to period. Wang et al. [46] combined TCS and link capacity improvement measure and proposed a biobjective bilevel programming model to balance economic growth and environmental management. Miralinaghi et al. [47] applied TCS to the management of morning commute congestion, considering loss aversion behavior in purchasing credits. Rather than in theory, there are also some works including a case study based on experiments [48-51]. All of these works provide good inspirations for this paper.

The above-mentioned works indicate that TCS is a flexible scheme that can be combined with many other management methods to achieve various targets of traffic management. Given the advantages of TCS over other transport policies in management, we believe that there is a booming future for the further development and real practice of TCS policy.

1.2. Research Objectives and Contributions. To summarize, the emerging CAV technologies have gained increasing popularity among researchers and policy-makers, and TCS have been identified as an innovative alternative to tackle transportation problems as well. It is predictable that there will be increasing CAVs on the road in the near future and road traffic will remain as a mixed state for both HDVs and CAVs for a long time. In such a situation, existent works about TCS based on the assumption that all travelers travel by HDVs and their route choice follows UE principle may be inconsistent with the actual case due to the differences in
HDV and CAV. Therefore, more applicable models need to be established to characterize the travelers' behaviors for mixed traffic in the context of TCS.

However, to the best of our knowledge, only Han and Cheng's $[52,53]$ works have been found to investigate TCS in an SUE modeling framework. Furthermore, there has not been any work combining the penetration of CAVs and implementation of TCS together and investigating their interactions in practice so far. This paper aims to fill this gap by studying the traffic equilibrium for mixed traffic flows of HDVs and CAVs under the management of TCS. The results could help enhance the applicability of TCS given the market penetration of CAVs and enable policy-makers to better understand the market and route choice behaviors in the case of mixed traffic.

This paper formulated the stochastic user equilibriums for mixed traffic flows under TCS as variational inequality (VI) problems with exogenous and endogenous CAV penetration rate, respectively. Then a modified Lagrangian dual method embedded with a revised Smith's routeswapping (RSRS) algorithm is proposed to solve the problems and it is proved to be able to converge to a local optimum. Finally, based on the example networks, the impacts of CAV penetration and its extra expense are investigated. The main contributions of this paper are twofold. First, the impacts of CAV penetration and its extra expense on network performance and credit market under TCS are demonstrated in the context of exogenous and endogenous CAV penetration rate, respectively. Second, a novel driveway management, autonomous vehicle/credit charge (AVCC) link, is put forward and analytically proved to be effective in the CAV management under TCS.

The remainder of this paper is organized as follows. In Section 2, we analyze the SUE conditions under TCS with exogenous CAV penetration rate. The impacts of CAV penetration and the deployment of AVCC links on network performance are investigated. In Section 3, the CAV penetration rate is assumed to be endogenous. We conduct the analysis in a similar fashion and further study the impacts of CAV extra expense on network performance. In Section 4, we present concluding remarks and recommendations for future research.

\section{Tradable Credit Scheme for Mixed Traffic Flow with Exogenous CAV Penetration Rate}

Consider a general network $G=(N, A)$, with a set $N$ of nodes and a set $A$ of directed links. Let $W$ denote the set of O-D pairs and $R^{w}$ the set of all routes connecting O-D pair $w$. For each O-D pair $w \in W$, travelers can travel on it by either HDVs or CAVs. Let $M$ denote the set of vehicle types, that is, $M=\{\mathrm{HDV}, \mathrm{CAV}\}$. In this section, we assume that travel demand is given and fixed, denoted by a vector $\mathbf{d}=\left(\mathbf{d}^{\mathrm{HDVT}}, \mathbf{d}^{\mathrm{CAVT} T}\right)^{T}, \quad$ where $\mathbf{d}^{\mathrm{HDV}}=\left\{\mathbf{d}_{w}^{\mathrm{HDV}}, w \in W\right\}$, $\mathbf{d}^{\mathrm{CAV}}=\left\{\mathbf{d}_{w}^{\mathrm{CAV}}, w \in W\right\}$, and $d_{w}^{m}$ is the travel demand of vehicle type $m$ within O-D pair $w$. The flow of vehicle type $m$ on route $r$ is denoted by $f_{r, w}^{m}$.

To determine the link travel times of CAVs and HDVs in the mixed flow, the Bureau of Public Roads (BPR) function is used. 


$$
t_{a}\left(v_{a}^{\mathrm{HDV}}, v_{a}^{\mathrm{CAV}}\right)=t_{a}^{0}\left(1+0.15\left(\frac{v_{a}^{\mathrm{HDV}}+v_{a}^{\mathrm{CAV}}}{C_{a}}\right)^{4}\right), \quad a \in A,
$$

where $t_{a}^{0}$ is the free flow travel time on link $a$; $v_{a}^{\mathrm{HDV}}$ and $v_{a}^{\mathrm{CAV}}$ represent the HDV and CAV flow on link $a$, respectively; $C_{a}$ represents the capacity of link $a$, namely,

$$
C_{a}\left(v_{a}^{\mathrm{HDV}}, v_{a}^{\mathrm{CAV}}\right)=\frac{1}{\left(\left(v_{a}^{\mathrm{HDV}} /\left(v_{a}^{\mathrm{HDV}}+v_{a}^{\mathrm{CAV}}\right)\right) \cdot\left(1 / C_{a}^{\mathrm{HDV}}\right)+\left(v_{a}^{\mathrm{CAV}} /\left(v_{a}^{\mathrm{HDV}}+v_{a}^{\mathrm{CAV}}\right)\right) \cdot\left(1 / C_{a}^{\mathrm{CAV}}\right)\right)}, \quad a \in A,
$$

where $C_{a}^{\mathrm{HDV}}$ and $C_{a}^{\mathrm{CAV}}$ represent the link capacity for pure HDV flow and CAV flow, respectively. This assumption was also adopted by Wang et al. [28], Liu and Song [54], Mehr and Horowitz [55], and Noruzoliaee et al. [56]. Substituting (2) into (1), we can get

$$
t_{a}\left(v_{a}^{\mathrm{HDV}}, v_{a}^{\mathrm{CAV}}\right)=t_{a}^{0}\left(1+0.15\left(\frac{v_{a}^{\mathrm{HDV}}}{C_{a}^{\mathrm{HDV}}}+\frac{v_{a}^{\mathrm{CAV}}}{C_{a}^{\mathrm{CAV}}}\right)^{4}\right), \quad a \in A
$$

It can be easily confirmed that the link travel time function defined above is strictly monotonic with respect to link flow of either HDVs or CAVs.

The total aggregate flow $v_{a}$ on link $a$ can be expressed in terms of route flows as follows:

$$
\begin{aligned}
v_{a}^{m} & =\sum_{w \in W} \sum_{r \in R^{w}} f_{r, w}^{m} \delta_{a, r}^{w}, \quad a \in A, m \in M, \\
v_{a} & =\sum_{m \in M} v_{a}^{m}, \quad a \in A,
\end{aligned}
$$

where $\delta_{a, r}^{w}=1$ if link $a$ belongs to route $r$ and 0 otherwise. The associations between route flows and travel demand are expressed as

$$
\begin{aligned}
\sum_{r \in R^{w}} f_{r, w}^{m} & =d_{w}^{m}, \quad w \in W, m \in M, \\
\sum_{m \in M} d_{w}^{m} & =d_{w}, \quad w \in W .
\end{aligned}
$$

For simplicity, we denote route and link flows in vectors as $\mathbf{f}=\left(\mathbf{f}^{\mathrm{HDVT}}, \mathbf{f}^{\mathrm{CAVT}}\right)^{\mathrm{T}}$, where $\mathbf{f}^{\mathrm{HDV}}=\left\{f_{r, w}^{\mathrm{HDV}}, r \in R^{w}, w \in W\right\}$ and $\mathbf{f}^{\mathrm{CAV}}=\left\{f_{r, w}^{\mathrm{CAV}}, r \in R^{w}, w \in W\right\}$. Then the set of all feasible route flow patterns $\left(\mathbf{f}^{\mathrm{HDV}}, \mathbf{f}^{\mathrm{CAV}}\right)$ can be defined by

$$
\Omega=\left\{\begin{array}{l|ll}
\left(\mathbf{f}^{\mathrm{HDV}}, \mathbf{f}^{\mathrm{CAV}}\right) & \begin{array}{ll}
v_{a}^{m}=\sum_{w \in W} \sum_{r \in R^{w}} f_{r, w}^{m} \delta_{a, r}^{w}, & f_{r, w}^{m} \geq 0, a \in A, r \in R^{w}, \\
\sum_{r \in R^{w}} f_{r, w}^{m}=d_{w}^{m}, \sum_{w \in W} d_{w}^{m}=d_{w}, & w \in W, m \in M .
\end{array}
\end{array}\right\}
$$

Under the tradable credit scheme discussed in this paper, credits are uniformly distributed among all the travelers, by which each traveler gets $k$ credits initially. Free trading of credits among travelers is allowed in the credit market. Let $K$ denote the total amount of credits distributed with $K=k \cdot \sum_{w \in W} d_{w}$. The link-specific but user-anonymous credit charging scheme is denoted by $\kappa=\left\{\kappa_{a}, a \in A\right\}$, where $\kappa_{a}$ is the credit charge for any traveler who uses link $a$. As in the work of Yang and Wang [32], we use $(K, \kappa)$ to characterize a credit charge scheme $\kappa$ under a total number of credits $K$ issued in the market.

It is obvious that not all credit schemes can guarantee the existence of feasible network flow patterns in a fixed demand case. The total supply of credits might be too low to meet the needs of all travelers going through the network even if all of them use the routes with the least charge. Thus, for a given vehicle type-specific O-D demand, we define the corresponding feasible set of credit schemes which ensures the existence of feasible network flow patterns. Let $\Psi$ denote the feasible set of credit schemes where

$$
\Psi=\left\{(K, \kappa) \mid \exists\left(\mathbf{f}^{\mathrm{HDV}}, \mathbf{f}^{\mathrm{CAV}}\right) \in \Omega \text { such that } \sum_{a \in A} \kappa_{a} v_{a} \leq K\right\}
$$

where $\Omega$ is defined by (7); and we assume that $\Psi$ is nonempty.

Besides, in the context of mixed traffic flows, we assume that the CAV penetration rate is exogenously determined in this section. Namely, the CAV penetration rate $p_{\mathrm{CAV}}$ is given and is constant during the analysis. The CAVs are assumed to be uniformly distributed to all the O-D pairs with the same penetration rate, i.e., 


$$
\frac{d_{w_{1}}^{\mathrm{CAV}}}{d_{w_{1}}}=\frac{d_{w_{2}}^{\mathrm{CAV}}}{d_{w_{2}}}=\frac{d_{w_{3}}^{\mathrm{CAV}}}{d_{w_{3}}}=\cdots=\frac{d_{|W|}^{\mathrm{CAV}}}{d_{|W|}}=p_{\mathrm{CAV}}
$$

where $|W|$ denotes the total number of O-D pairs.

\subsection{Stochastic User Equilibrium for Mixed Traffic Flow.} Based on the assumptions above, the travel cost for each traveler consists of two parts: (1) travel time cost and (2) credit cost. To characterize the utility of using the two types of vehicles, different values of time (VOT) are applied. Previous works show that CAV users tend to have lower VOT because they can spend the time during travel on other man beings due to the high level of automation of CAVs [57]. The travel cost for user class $m$ using route $r$ between $\mathrm{O}-\mathrm{D}$ pair $w$ is given by

$$
\begin{array}{r}
c_{r, w}^{m}=\sum_{a \in A}\left(\beta_{m} t_{a}\left(v_{a}^{\mathrm{HDV}}, v_{a}^{\mathrm{CAV}}\right)+p \kappa_{a}\right) \delta_{a, r}^{w}, \\
r \in R^{w}, w \in W, m \in M,
\end{array}
$$

where $\beta_{m}$ denotes the average VOT for vehicle type $m ; p$ denotes the unit credit price. The credit cost for using a route $r \in R^{w}$ is given by the credit charge multiplied by the unit credit price, $p$.

In this work, both HDVs and CAVs are assumed to follow the SUE principle. Namely, at equilibrium, no user can unilaterally change his path to reduce the perceived travel cost. Nonetheless, we assume that CAV users have much lower perception variances than HDV users on travel cost since CAVs can obtain information on traffic conditions through vehicle-to-infrastructure and vehicle-to-vehicle communications. Thereby, CAVs are more likely to choose paths with the minimum travel cost for the corresponding O-D pairs compared with HDVs.

To characterize the equilibrium condition, a logit-based model is applied in this section. Let $U_{r, w}^{m}$ denote the disutility for vehicle type $m$ traveling on route $r$ connecting O-D pair $w$, formulated as

$$
U_{r, w}^{m}=c_{r, w}^{m}+\xi_{r, w}^{m}, \quad r \in R^{w}, w \in W, m \in M,
$$

where $\xi_{r, w}^{m}, m \in M$ are independent and identical distributed Gumbel variables with zero mean. According to the utility maximization theory, the logit-based SUE condition is formulated as

$$
f_{r, w}^{m}=d_{w}^{m} \cdot P_{r, w}^{m}, \quad r \in R^{w}, w \in W, m \in M .
$$

The route choice probability for vehicle type $m$ user traveling on O-D pair $w$ is governed by the following logit formulae:

$$
P_{r, w}^{m}=\frac{\exp \left(-\theta_{m} c_{r, w}^{m}\right)}{\sum_{k \in R^{w}} \exp \left(-\theta_{m} c_{k, w}^{m}\right)}, \quad r \in R^{w}, w \in W, m \in M,
$$

where $\theta_{m}$ is the dispersion parameter to measure the degrees of travelers' perception errors of vehicle type $m$ user. A higher $\theta_{m}$ value means a smaller perception variation. It can be applied to demonstrate the quality of the provided traffic information. For the two typical vehicle type users, $\theta_{\mathrm{HDV}}<\theta_{\mathrm{CAV}}$ holds, which claims that the CAV users have lower perception variances on travel cost than HDV users.

The credit market equilibrium (ME) conditions are given by

$$
\begin{aligned}
& \sum_{a \in A} \kappa_{a} v_{a}^{*}=K, \quad \text { if } p^{*}>0, \\
& \sum_{a \in A} \kappa_{a} v_{a}^{*} \leq K, \quad \text { if } p^{*}=0 .
\end{aligned}
$$

Equations (14) and (15) represent the credit market clearing conditions, which imply that the equilibrium credit price is positive only if all the issued credits are consumed. In other words, the credit scheme will be nullified due to the worthless credit if there are remaining credits among the travelers.

2.2. Variational Inequality Formulation and Its Properties. With the assumptions given above, we now formulate the SUE for mixed traffic flows as a variational inequality (VI) problem and then demonstrate some essential properties of it.

The VI formulation is given by

$$
\sum_{w \in W} \sum_{r \in R^{w}} \sum_{m \in M}\left(c_{r, w}^{m^{*}}+\frac{1}{\theta_{m}} \ln f_{r, w}^{m^{*}}\right)\left(f_{r, w}^{m}-f_{r, w}^{m^{*}}\right) \geq 0, \quad \forall\left(\mathbf{f}^{\mathrm{HDV}}, \mathbf{f}^{\mathrm{CAV}}\right) \in \widetilde{\Omega}(K, \boldsymbol{\kappa}),
$$

where

$$
\widetilde{\Omega}(K, \boldsymbol{\kappa})=\left\{\left(\mathbf{f}^{\mathrm{HDV}}, \mathbf{f}^{\mathrm{CAV}}\right) \mid \sum_{a \in A} \kappa_{a} v_{a} \leq K, \quad\left(\mathbf{f}^{\mathrm{HDV}}, \mathbf{f}^{\mathrm{CAV}}\right) \in \Omega\right\} \in \Omega .
$$

First, we are here to prove that the above VI problem is equivalent to the SUE conditions.
Proposition 1. The solution to the VI problem (16) is equivalent to the stochastic user equilibrium conditions (12) and (13). 
Proof. See Appendix A.

For convenience, let $C_{r, w}^{m}=c_{r, w}^{m}+\left(1 / \theta_{m}\right) \ln f_{r, w}^{m}$ and let $\mathbf{C}^{\mathrm{HDV}}$ and $\mathbf{C}^{\mathrm{CAV}}$ be the vectors of $\left\{C_{r, w}^{\mathrm{HDV}}\right\}$ and $\left\{C_{r, w}^{\mathrm{CAV}}\right\}$, respectively.

Then, we are going to discuss the existence of solutions to the VI problem.

Proposition 2. At least one solution of the VI problem (11) exists.

Proof. Since the feasible set $\widetilde{\Omega}(K, \kappa)$ is compact and convex, given a credit scheme $(K, \kappa) \in \Psi$ and the travel cost of HDV and $\mathrm{CAV}$ users, both $\mathrm{C}^{\mathrm{HDV}}$ and $\mathrm{C}^{\mathrm{CAV}}$ are continuous with respect to route flow pattern $\left(\mathbf{f}^{\mathrm{HDV}}, \mathbf{f}^{\mathrm{CAV}}\right)$. According to Facchinei and Pang [58], it can be proved that at least one solution $\left(\mathbf{f}^{\mathrm{HDV}}, \mathbf{f}^{\mathrm{CAV}}\right)$ satisfies the VI problem (16).

Let $\mathbf{C}=\left(\mathbf{C}^{\mathrm{HDVT}}, \mathbf{C}^{\mathrm{CAVT}}\right)^{T}$. Proposition 2 implies that there exists at least one route flow pattern satisfying VI problem (16). However, since the link travel time is asymmetric between HDVs and CAVs, the generalized travel cost vector $\mathbf{C}$ is asymmetric to path flow $\mathbf{f}$. Thus, the VI problem may not be monotonic due to the asymmetric relationship and cause a failure to achieve a global convergence [28]. To circumvent this difficulty, we apply a route-swapping-based algorithm to solve VI problem (16). This algorithm is designed to find a stable local convergence of the path flow rather than global convergence. Some researchers successfully applied route-swapping-based algorithm to a logitbased SUE problem even when the monotonicity of the travel cost is not met $[59,60]$. Hence the nonmonotonicity can be tackled with an RS-based algorithm. The routeswapping-based algorithm applied in this paper will be introduced later in Section 2.3.

Apart from the flow patterns, under a tradable credit scheme, the uniqueness of credit price is a critical factor contributing to the stability and predictability of the credit market. Thus, it is necessary to establish the conditions for unique equilibrium credit price.

Proposition 3. Given a tradable credit scheme $(K, \kappa) \in \Psi$, with an equilibrium route flow pattern $\left(\mathbf{f}^{\mathrm{HDV}}, \mathrm{f}^{\mathrm{CAV}}\right)$ the equilibrium credit price $p^{*}$ is unique if the following condition is satisfied: among all the corresponding SUE path flow patterns, there exists at least one of the typical vehicle users (HDV users or CAV users) whose equilibrium path set always contains the same two (or more) paths connecting one O-D pair with different credit charges.
Proof. Accordingly, it is assumed that, for any $\left(\mathbf{f}^{\mathrm{HDV}}, \mathbf{f}^{\mathrm{CAV}}\right) \in \widetilde{\Omega}(K, \kappa)$, the equilibrium path set of a user class $m$ always contains two paths, $r_{1}, r_{2} \in R^{w}$, connecting O-D pair $w$ and $\kappa_{r_{1}, w} \neq \kappa_{r_{2}, w}$, where $\kappa_{r, w}=\sum_{a \in A} \kappa_{a} \delta_{a, r}^{w}$. From SUE condition (7), we have

$$
\frac{f_{r_{1}, w}^{m^{*}}}{f_{r_{2}, w}^{m^{*}}}=\frac{\exp \left(-\theta_{m} c_{r_{1}, w}^{m^{*}}\right)}{\exp \left(-\theta_{m} c_{r_{2}, w}^{m^{*}}\right)}=\frac{\exp \left(-\theta_{m} t_{r_{1}, w}^{*}-\theta_{m} p^{*} \kappa_{r_{1}, w}\right)}{\exp \left(-\theta_{m} t_{r_{2}, w}^{*}-\theta_{m} p^{*} \kappa_{r_{2}, w}\right)},
$$

where $t_{r, w}^{*}=\sum_{a \in A} t_{a}^{*} \delta_{a, r}^{w}$. Then it follows readily from (18) that

$$
p^{*}=\frac{\left(\ln f_{r_{1}, w}^{m^{*}}-\ln f_{r_{2}, w}^{m^{*}}\right)+\theta_{m}\left(t_{r_{1}, w}^{*}-t_{r_{2}, w}^{*}\right)}{\theta_{m}\left(\kappa_{r_{2}, w}-\kappa_{r_{1}, w}\right)} .
$$

Therefore, the credit price at market equilibrium is uniquely determined.

2.3. Solution Algorithm. Due to the asymmetric impacts of HDVs and CAVs on link travel cost and the complexity of multiclass SUE problem, the traditional approach such as the Method of Successive Average (MSA) cannot be used to solve the proposed model for mixed traffic flow. Instead, a modified Lagrangian dual method embedded with a routeswapping-based solution algorithm is applied in this paper.

2.3.1. Revised Smith's Route-Swapping Algorithm. Studies have shown that route-swapping models are able to obtain a feasible solution algorithm for either UE [61, 62] or SUE $[59,60]$ network equilibrium problems. To overcome the overestimation of projected route-swapping (PRS) algorithm [61] on the least-cost path flows and resulting slow convergence, Wang et al. [28] developed a revised Smith's route-swapping (RSRS) algorithm and proved that it can converge much faster than PRS algorithm.

In the RSRS algorithm, both the HDV and CAV path flows $\mathbf{f}_{n+1}^{\mathrm{HDV}}$ and $\mathbf{f}_{n+1}^{\mathrm{CAV}}$ in iteration $n+1$ will be updated according to the following equations:

$$
\mathbf{f}_{n+1}=\mathbf{f}_{n}+\alpha_{n} \boldsymbol{\Phi}\left(\mathbf{f}_{n}\right)=\left[\begin{array}{l}
\mathbf{f}_{n}^{\mathrm{HDV}} \\
\mathbf{f}_{n}^{\mathrm{CAV}}
\end{array}\right]+\alpha_{n}\left[\begin{array}{l}
\boldsymbol{\Phi}^{\mathrm{HDV}}\left(\mathbf{f}_{n}\right) \\
\boldsymbol{\Phi}^{\mathrm{CAV}}\left(\mathbf{f}_{n}\right)
\end{array}\right],
$$

where $\alpha_{n}>0$ is a predetermined step size; $\Phi^{\mathrm{HDV}}\left(\mathbf{f}_{n}\right)=$ $\left\{\Phi_{r, w}^{\mathrm{HDV}}, \forall r \in R^{w}, w \in W\right\}$ and $\Phi^{\mathrm{CAV}}\left(\mathbf{f}_{n}\right)=\left\{\Phi_{r, w}^{\mathrm{CAV}}, \forall r \in R^{w}\right.$, $w \in W\}$ are updated according to

$$
\Phi_{r, w}^{m}\left(\mathbf{f}_{n}\right)=\sum_{k \in R^{w}}\left[f_{k, w}^{m}(n)\left(C_{k, w}^{m}\left(\mathbf{f}_{n}\right)-C_{r, w}^{m}\left(\mathbf{f}_{n}\right)\right)_{+}-f_{r, w}^{m}(n)\left(C_{r, w}^{m}\left(\mathbf{f}_{n}\right)-C_{k, w}^{m}\left(\mathbf{f}_{n}\right)\right)_{+}\right], \quad r \in R^{w}, w \in W, m \in M
$$

where $f_{r, w}^{m}(n)$ denotes the flow of vehicle type $m$ on route $r$ connecting O-D pair $w$ in iteration $n$. $\left(C_{k, w}^{m}\left(\mathbf{f}_{n}\right)-\right.$ $\left.C_{k, w}^{m}\left(\mathbf{f}_{n}\right)\right)_{+}=C_{k, w}^{m}\left(\mathbf{f}_{n}\right)-C_{k, w}^{m}\left(\mathbf{f}_{n}\right) \quad$ if $\quad C_{k, w}^{m}\left(\mathbf{f}_{n}\right)>C_{r, w}^{m}\left(\mathbf{f}_{n}\right)$; otherwise $\left(C_{k, w}^{m}\left(\mathbf{f}_{n}\right)-C_{r, w}^{m}\left(\mathbf{f}_{n}\right)\right)_{+}=0$.
This algorithm can converge much faster because it avoids solving the subproblem in each iteration. Instead, it determines the descent direction quickly based on the path flow and generalized travel cost acquired from the previous 
iteration. Additionally, the RSRS algorithm can increase flows not only on routes with the least cost increase but also on other routes with relatively low cost; this further expedites the convergence when the solution is close to the optimum [28].

The choice of the predetermined step size $\alpha_{n}$ at iteration $n$ can refer to the strategy proposed by Huang and Lam [61]. It assumed that the step sizes are the same for a large number of consecutive iterations and nonincreasing with respect to the number of iterations. Namely, the step size can be formulated as

$$
\alpha_{n}=e_{1} \frac{1}{\left[\left(n / e_{2}\right)+1\right]},
$$

where $e_{1}$ is a small positive value and $e_{2}$ is the number of iterations within which the step sizes are the same. $\left[\left(n / e_{2}\right)+\right.$ $1]$ denotes the integer part of $\left(n / e_{2}\right)+1$. The convergence criterion of RSRS algorithm is given by

$$
G=\frac{\sum_{w \in W} \sum_{r \in R^{w}} \sum_{m \in M} f_{r, w}^{m}\left(C_{r, w}^{m}-C_{\min , w}^{m}\right)}{\sum_{w \in W} \sum_{r \in R^{w}} \sum_{m \in M} f_{r, w}^{m} C_{r, w}^{m}} .
$$

2.3.2. Modified Lagrangian Dual Method. The RSRS algorithm introduced above is embedded as an inner iteration in a modified Lagrangian dual (MLD) method. The MLD method as outer iteration was proposed by Han and Cheng [52]. This method is designed to find the equilibrium credit price under a given TCS. Applying this method, the unit credit price is initialized as a positive number and will be updated till it is sufficiently close to its optimum and correctly reflects the market equilibrium.

Combining the MLD method and RSRS algorithm, the iterative process is given as follows.

Step 0. Determine the constant of tolerances $G_{0}$ and $\varepsilon_{p}$ and the sequence of move sizes $\alpha_{n}$ given by (22). Randomly initialize the credit price $p_{i}>0$. Set $i:=1$.

Step 1. Run an RSRS algorithm with $p_{i}$ :

(i) Initialization: set $n:=1$. Assign the O-D demands of HDVs and CAVs uniformly to all the routes connecting the O-D pair. Denote the resulting route flows for HDVs and CAVs as $\mathbf{f}_{n}^{\mathrm{HDV}}$ and $\mathbf{f}_{n}^{\mathrm{CAV}}$, respectively.

(ii) Generalized travel cost update: given the credit price $p_{i}$, update the generalized travel cost $\mathbf{C}$ by (19).

(iii) Route flow update: update route flows for HDVs and CAVs according to (20).

(iv) Convergence check: if the convergence criterion,

$$
G \leq G_{0}
$$

is satisfied, stop the RSRS algorithm and set $\mathbf{f}^{\mathrm{HDV}}=\mathbf{f}_{n+1}^{\mathrm{HDV}}$ and $\mathbf{f}^{\mathrm{CAV}}=\mathbf{f}_{n+1}^{\mathrm{CAV}}$. Otherwise, set $n:=n+1$ and go to (ii).

Step 2. Check the scheme validity. If $\sum_{a \in A}$ $\kappa_{a} v_{a}\left(\mathbf{f}^{H D V}, \mathbf{f}^{\mathrm{CAV}}\right)<K$, set $p_{i+1}=0$. Otherwise, set the credit price $p_{i+1}$ with $p_{i+1}=P_{+}\left(p_{i}+(1 / i) L^{\prime}(p)\right)$.
Step 3. If the convergence criterion,

$$
\left|\left(p_{i+1}-p_{i}\right)\right| \leq \varepsilon_{p},
$$

is satisfied, stop the algorithm and return $p^{*}=p_{i+1}$ and $\left(\mathbf{f}^{\mathrm{HDV}}, \mathbf{f}^{\mathrm{CAV}}\right)$. Otherwise, set $i:=i+1$ and go to Step 1 . Meanwhile, $L^{\prime}(p)$ denotes the derivative of Lagrangian function defined in equation (A.1) with respect to $p$. The notation $P_{+}$is a nonnegative projection operator defined by

$$
P_{+}(y)=\max \left\{0, \arg \min _{x \in R^{+}}\|y-x\|^{2}\right\} .
$$

Hence, $p_{i+1}$ in Step 2 can be calculated by

$$
\begin{aligned}
p_{i+1}= & P_{+}\left(p_{i}+\frac{1}{i}\left(\sum_{a \in A} \kappa_{a} v_{a}\left(\mathbf{f}^{\mathrm{HDV}}, \mathbf{f}^{\mathrm{CAV}}\right)-K\right)\right) \\
& =\max \left(0, p_{i}+\frac{1}{i}\left(\sum_{a \in A} \kappa_{a} v_{a}\left(\mathbf{f}^{\mathrm{HDV}}, \mathbf{f}^{\mathrm{CAV}}\right)-K\right)\right) .
\end{aligned}
$$

As mentioned before, the MLD method is performed as the outer iteration to update the unit credit price. In each outer iteration, an inner iteration of RSRS algorithm proceeds to update the optimal path flow patterns. The procedure will be terminated after the difference between two successive credit prices is within a tolerance. The proof of the convergence of the outer MLD method and inner RSRS algorithm can follow the same fashions proposed by Han and Cheng [52] and Wang et al. [28]. We omit the proof here to avoid duplication. The convergence results will be shown in the following subsection.

2.4. Numerical Analysis. Based on the algorithm proposed in the last section, we are able to solve the VI problem (16). In this section, we adopt a small network to explore the impacts of exogenous CAV penetration on the network under TCS.

As shown in Figure 1, the example network consists of six nodes, seven links, two O-D pairs:, O-D pair 1 (node 1 $\longrightarrow$ node 2 ) and O-D pair 2 (node $3 \longrightarrow$ node 4 ), and four routes: route 1 (link 1), route 2 (link 2-5-6), route 3 (link 4-57 ), and route 4 (link 3 ). The values of time for HDV and CAV users are $\beta_{\mathrm{HDV}}=5, \beta_{\mathrm{CAV}}=2.5$. The information of the network and credit scheme is given in Table 1 .

The travel demands are 60 units for O-D pair 1 and 50 units for $\mathrm{O}-\mathrm{D}$ pair 2 , respectively. The dispersion parameters of perception errors are given as $\theta_{\mathrm{HDV}}=0.01$ and $\theta_{\mathrm{CAV}}=0.99 \theta=1.00 \theta_{\mathrm{CAV}}$. We assume that the total amount of distributed credits is 660 , and each traveler on the network is initially distributed 6 credits.

The parameters for the proposed solution algorithm are $e_{1}=0.1, e_{2}=10, G_{0}=0.01$ and $\varepsilon_{p}=0.01$. The convergence results for the algorithm are presented in Figure 2. It can be seen that it converges very fast in the first 10 iterations and takes about 80 iterations to achieve the objective values. 


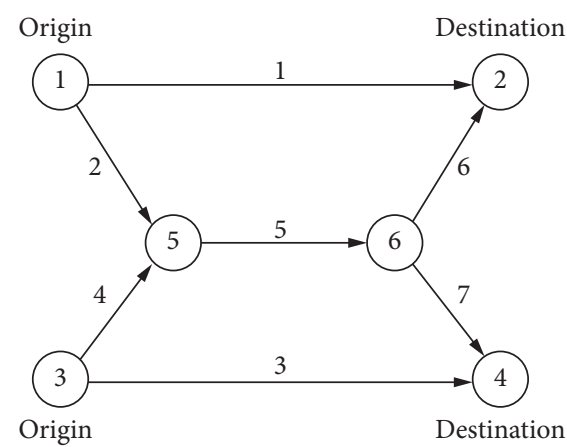

FIgURE 1: The small network.

TABLE 1: Input data of the small network.

\begin{tabular}{lcccc}
\hline Link & $\begin{array}{c}\text { Free flow travel } \\
\text { time }\end{array}$ & $\begin{array}{c}\text { Capacity for } \\
\text { HDV }\end{array}$ & $\begin{array}{c}\text { Capacity for } \\
\text { CAV }\end{array}$ & $\begin{array}{c}\text { Credit } \\
\text { charge }\end{array}$ \\
\hline 1 & 10 & 35 & 70 & 9 \\
2 & 3 & 30 & 60 & 2 \\
3 & 12 & 35 & 70 & 8 \\
4 & 4 & 35 & 70 & 1 \\
5 & 5 & 35 & 70 & 3 \\
6 & 3 & 35 & 70 & 2 \\
7 & 4 & 25 & 50 & 1 \\
\hline
\end{tabular}

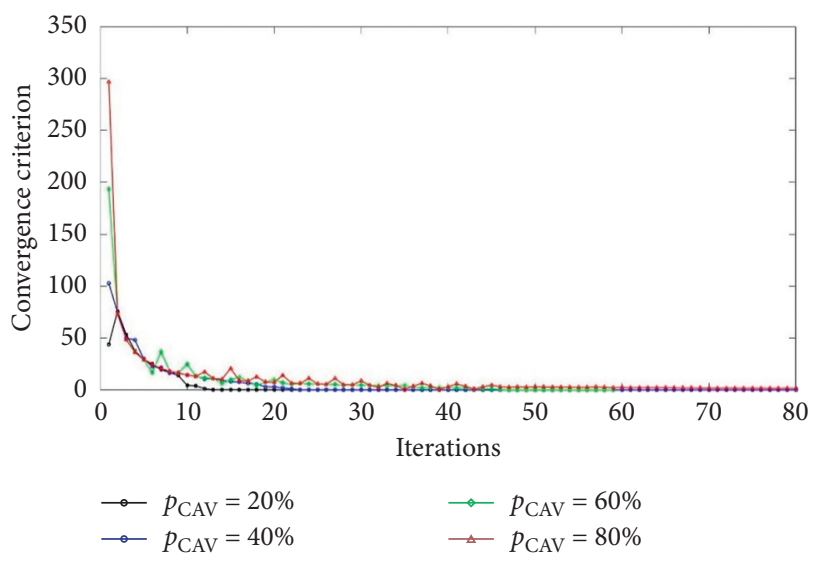

FIGURE 2: Convergence results for unit credit price under various CAV penetration rates.

2.4.1. The Deployment of AVCC Links. To seek whether there is an efficient and practical approach to manage CAVs under a TCS, a novel driveway management is proposed in this paper. Liu and Song [54] proposed a new form of managed lanes for CAVs, designated as autonomous vehicle/toll lanes, which grant free access to CAVs while allowing HDVs to access the lanes by paying a toll. In the context of TCS, a similar form of lanes, autonomous vehicle/credit charge (AVCC) lanes, can be applied to the management of CAVs. Accordingly, it is free for CAVs while charging credits for HDVs.

Liu and Song [54] and Wang et al. [28] have demonstrated that an AV-dedicated lane can reduce the network performance when the CAV penetration rate is low.
Compared with AV-dedicated lane, an AVCC lane is theoretically more flexible, since the space resources will not be wasted even there are few CAVs on the road.

Due to the lane-changing behaviors caused by overtaking and turning, charging credit is practically difficult if an AVCC lane is set on the link. Thus, AVCC link rather than AVCC lane is considered in this paper. Once the AVCC link is deployed under a given TCS, the credit charge scheme will be no longer vehicle type-anonymous. Thus, we define $\kappa_{a}^{m}$ as the credit charge for vehicle type $m$ using link $a \in A$. $c_{r, w}^{m}=\sum_{a \in A}\left(t_{a}\left(v_{a}^{\mathrm{HDV}}, v_{a}^{\mathrm{CAV}}\right)+p \kappa_{a}^{m}\right) \delta_{a, r}^{w}, \quad r \in R^{w}, w \in W, m \in M$.

To investigate the impact of the deployment of AVCC links, link 5 in the small network is converted to an AVCC link which charges credits for HDVs and is free for CAVs. The main findings are given in the next subsection.

2.4.2. Main Findings. First, we try to examine whether a TCS can improve the performance of the network with mixed traffic flows. Figure 3 shows the improvement on travel time before and after the implementation of TCS. The network without implementation of TCS is regarded as the benchmark and the degree of being better off denotes the ratio of the travel time reduction to the travel time before implementing TCS.

From Figure 3(a), it can be observed that both HDV and CAV users can benefit from the implementation of TCS. The degree of being better off for each vehicle type increases monotonically as the CAV penetration rate increases. This is because the penetration of CAVs can increase the link capacity to reduce travel time.

After the deployment of AVCC link, it can be seen from Figure 3(b) that the degree of being better off for all users is larger than that without AVCC link (except when $p_{\mathrm{CAV}}=0$, since AVCC link does not work its way when there is no $\mathrm{CAV}$ in the network), which implies the deployment of AVCC link can improve the efficiency of a given TCS.

Additionally, it is interesting to note that CAV users benefit much more than HDV users compared with the case in Figure 3(a), especially when the CAV penetration is low. This is mainly due to the "guidance" from the AVCC link to make CAVs get together. Specifically, there will be a considerable part of CAVs previously using link 1 and link 2 switching to link 5 after the latter is converted to an AVCC link. Correspondingly, there will be some HDVs previously on link 5 switching to link 1 or link 3 . This leads to an enhancement on capacity of link 5 and thus a reduction on CAV's average travel time. When the CAV penetration is high, the benefit of enhanced capacity will be also gained by the minority HDVs, so that the advantage of CAV is weakened and the travel time gap is shortened.

To further figure out the reason for improving the efficiency of TCS, we then focus on the behavior of HDVs. Figure 4 shows the travel cost gap for HDV users on the two O-D pairs before and after the deployment of AVCC link. Since each O-D pair has two routes, the travel cost gap 


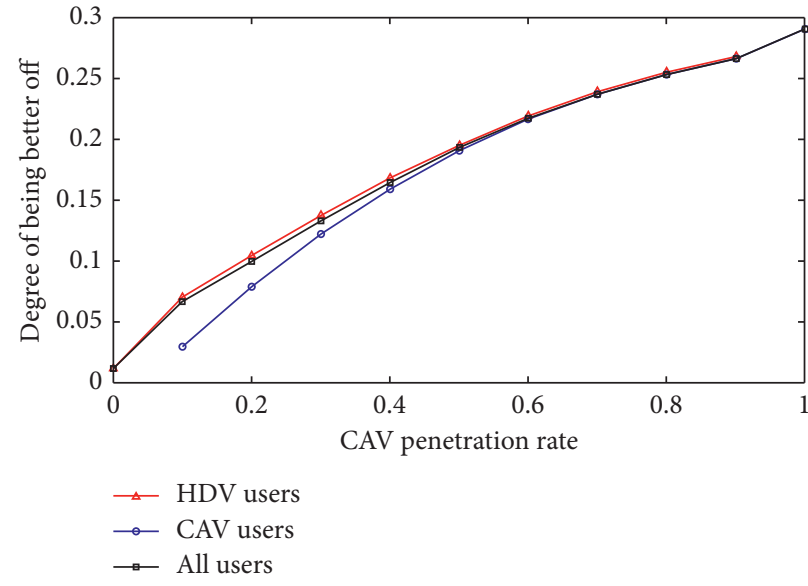

(a)

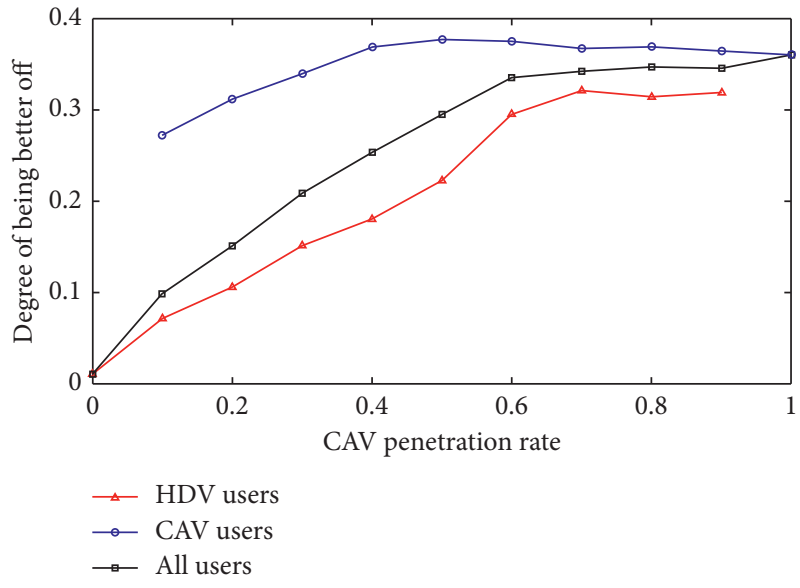

(b)

FIGURE 3: Improvement on network performance under tradable credit scheme. (a) Degree of being better off without AVCC link. (b) Degree of being better off with AVCC link.

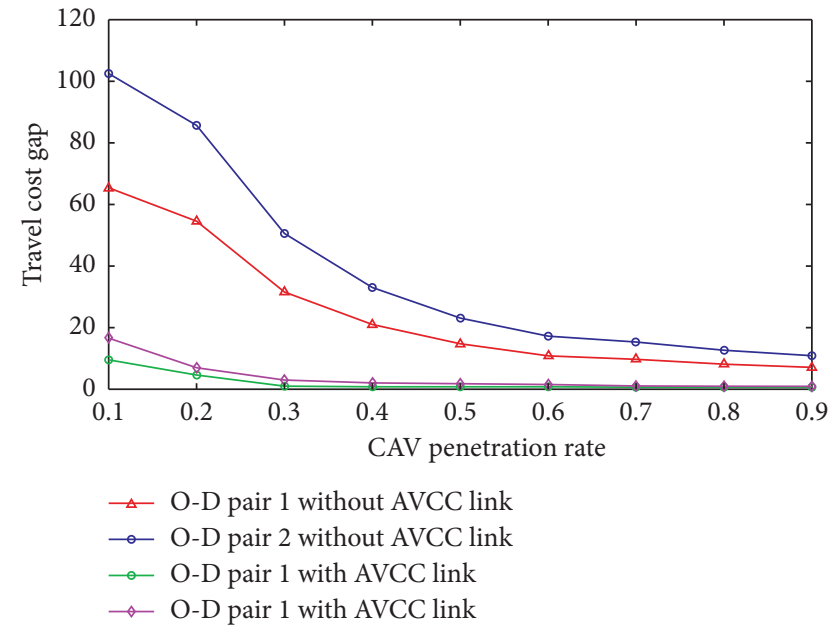

Figure 4: Travel cost gap for HDV users on each O-D pair.

represents the difference between route 1 and route 2 for $\mathrm{O}-\mathrm{D}$ pair 1 and that between route 3 and route 4 for O-D pair 2 .

From Figure 4, we can observe that the travel cost gaps shrink as CAV penetration increases. This can be explained by the dominance of UE rather than SUE effects for CAVs when the proportion of CAV increases. As mentioned before, with $\theta_{\mathrm{CAV}}$ approximated to 1.00 , we can say that the CAV users have a full information of the network and make their choices nearly based on the UE principle. In contrast, there are larger perception variances on travel cost for HDV users and they will not necessarily choose the route with actual least cost but choose the route with perceived least cost, that is, following SUE principle. This principle makes it possible for HDV users to choose the route with higher travel cost and gives rise to the different actual travel costs for the routes between each O-D pair. The actual travel cost gap between the routes can be considerably large when HDVs occupy the majority of vehicles. However, when CAVs occupy the majority, the SUE effect will be weakened and the actual travel cost gap will be smaller.
It can be also seen that the deployment of AVCC link can shrink the travel cost gap between the two alternative routes, particularly when the CAV penetration is low. This implies that the AVCC link can improve the efficiency of TCS because it can reduce the actual travel cost gap between the two routes connecting an O-D pair and make the distribution of HDVs more balanced. In other words, the improvement brought by the deployment of AVCC link is rooted in the better guidance for the HDVs with high perception variance to choose the routes with lower actual travel cost. It partially alleviates the imbalanced distribution caused by perception variance and thus leads to a better performance of the network.

Moreover, the impact of CAV penetration on credit market is investigated by examining the variations in equilibrium credit price and trading volume of credits, as shown in Figure 5. It can be seen that the credit price goes down as the CAV penetration rate increases. It should be noted that the deployment of AVCC link can further lower the credit price. In terms of the trading volume in the credit market, we can see that the deployment of AVCC links not only extensively increases the trading volume but also gives rise to a positive correlation between CAV penetration rate and trading volume. This is because, with AVCC links, CAV users need to pay less credits and they could sell their unused credits to HDV users to gain benefits, which stimulates that credit-trading among travelers leads to an increase in trading volume. Also, as CAV penetration increases, more CAV users sell the credits to HDV users and further facilitate an increase in trading volume.

\section{Tradable Credit Scheme for Mixed Traffic Flow with Endogenous CAV Penetration Rate}

3.1. Stochastic User Equilibrium for Mixed Traffic Flow. In this section, we assume that the CAV penetration rate is endogenously determined by the traveler's willingness to use a CAV. A nested-logit model is applied to describe 


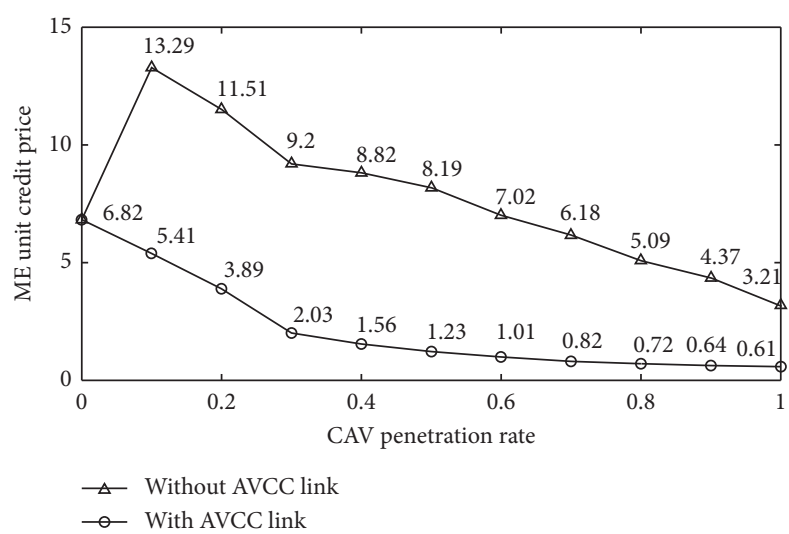

(a)

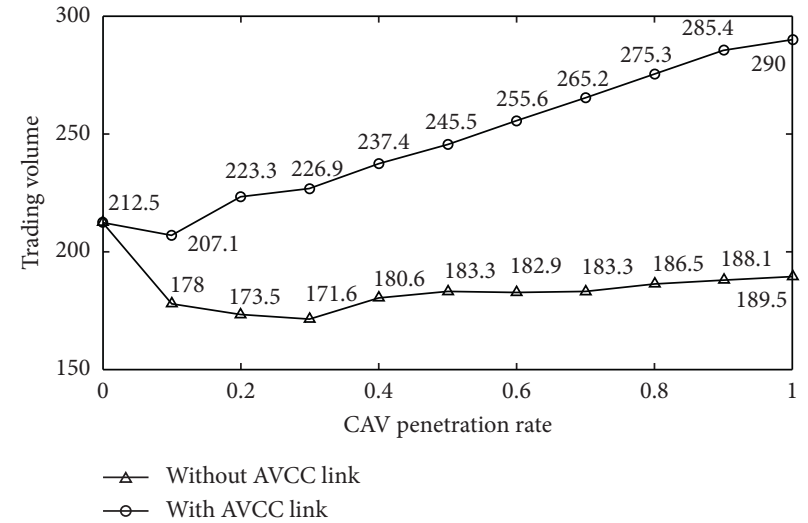

(b)

FIgURE 5: States of credit market under various CAV penetration rates. (a) Market equilibrium unit credit price. (b) Trading volume among travelers.

travelers' choices of vehicle types and routes, where travelers first decide whether to travel by HDV or CAV based on the expected utility and then choose the routes with minimized perceived travel disutility. The travel costs for HDV and CAV users using route $r$ between O-D pair $w$ are given by

$$
\begin{aligned}
& c_{r, w}^{\mathrm{HDV}}=\sum_{a \in A}\left(\beta_{\mathrm{HDV}} t_{a}\left(v_{a}^{\mathrm{HDV}}, v_{a}^{\mathrm{CAV}}\right)+p \kappa_{a}\right) \delta_{a, r}^{w}, \quad r \in R^{w}, w \in W, \\
& c_{r, w}^{\mathrm{CAV}}=\sum_{a \in A}\left(\beta_{\mathrm{CAV}} t_{a}\left(v_{a}^{\mathrm{HDV}}, v_{a}^{\mathrm{CAV}}\right)+p \kappa_{a}\right) \delta_{a, r}^{w}+c_{0}, \quad r \in R^{w}, w \in W,
\end{aligned}
$$

where $c_{0}$ represents the CAV extra expenses (for purchasing an $\mathrm{CAV}$, acquiring an $\mathrm{AV}$ license, etc.) that are averagely distributed to each travel. Accordingly, the disutility for choosing vehicle type $m$ and traveling on route $r \in R^{w}$ is formulated as follows:

$$
U_{r, w}^{m}=c_{r, w}^{m}+\zeta_{r, w}^{m}, \quad r \in R^{w}, w \in W, m \in M,
$$

where $\zeta_{r, w}^{m}$ is also a random error representing unobservable factors. The SUE condition is formulated as

$$
f_{r, w}^{m}=d_{w} \cdot P_{r, w}^{m}, \quad r \in R^{w}, w \in W, m \in M .
$$

In the nested-logit model, the probability of choosing vehicle type $m$ traveling on route $r$ can be written as the product of the marginal probability $P_{w}(m)$ and conditional probability $P_{w}(k \mid m)$.

$$
P_{r, w}^{m}=P_{w}(k \mid m) \cdot P_{w}(m)=\frac{\exp \left(-\theta_{m} c_{r, w}^{m}\right)}{\sum_{k \in R^{w}} \exp \left(-\theta_{m} c_{k, w}^{m}\right)} \cdot \frac{\exp \left(-\bar{c}_{w}^{m}\right)}{\sum_{m^{\prime} \in M^{\prime}} \exp \left(-\bar{c}_{w}^{m^{\prime}}\right)}, \quad r \in R^{w}, w \in W, m \in M
$$

The term $\bar{c}_{r, w}^{m}$ given by equation (A.12) denotes the expected minimum perceived travel cost of vehicle type $m$ on O-D pair $w$.
3.2. Variational Inequality Formulation and Its Properties. Mathematically, the equilibrium conditions discussed above can be formulated as an equivalent VI problem as follows.

$$
\sum_{w \in W} \sum_{r \in R^{w}} \sum_{m \in M}\left(c_{r, w}^{m^{*}}+\frac{1}{\theta_{m}} \ln f_{r, w}^{m^{*}}\right)\left(f_{r, w}^{m}-f_{r, w}^{m^{*}}\right)+\sum_{w \in W} \sum_{m \in M}\left(1-\frac{1}{\theta_{m}}\right) \ln d_{w}^{m^{*}}\left(d_{w}^{m}-d_{w}^{m^{*}}\right) \geq 0, \quad \forall\left(\mathbf{f}^{\mathrm{HDV}}, \mathbf{f}^{\mathrm{CAV}}\right) \in \widetilde{\Omega}(K, \kappa)
$$

It is additionally subject to the nonnegativity and conservation constraints on demand of vehicle type $m$. 


$$
\begin{gathered}
d_{w}^{m} \geq 0, \quad w \in W, m \in M, \\
\sum_{m \in M} d_{w}^{m}=d_{w}, \quad w \in W .
\end{gathered}
$$

Proposition 4. The solution to the VI problem (33) is equivalent to the stochastic user equilibrium conditions (31) and (32).

Proof. See Appendix B.

Proposition 5. At least one solution of the VI problem (33) exists.

Proof. Let $\mathbf{H}^{\mathrm{HDV}}$ and $\mathbf{H}^{\mathrm{CAV}}$ be the vectors of $\{(1-(1 /$ $\left.\left.\left.\theta_{\mathrm{HDV}}\right)\right) \ln d_{w}^{\mathrm{HDV}}\right\}$ and $\left\{\left(1-\left(1 / \theta_{\mathrm{CAV}}\right)\right) \ln d_{w}^{\mathrm{HDV}}\right\}$. Since both $\mathbf{H}^{\mathrm{HDV}}$ and $\mathbf{H}^{\mathrm{CAV}}$ are continuous with respect to travel demand ( $\mathbf{d}^{\mathrm{HDV}}, \mathbf{d}^{\mathrm{CAV}}$ ), based on Proposition 2, it can be easily proved that at least one solution $\left(\mathbf{f}^{\mathrm{HDV}}, \mathbf{f}^{\mathrm{CAV}}, \mathbf{d}^{\mathrm{HDV}}, \mathbf{d}^{\mathrm{CAV}}\right)$ satisfies the VI problem (33).

Let $\mathbf{H}=\left(\mathbf{H}^{\mathrm{HDVT}}, \mathbf{H}^{\mathrm{CAVT}}\right)^{T}$. Since the vector $\mathbf{H}$ is monotonic with respect to $\mathbf{d}$, it will not affect the local convergence of the RSRS algorithm.

Then the condition for unique equilibrium credit price can be established as follows.

Proposition 6. Given a tradable credit scheme $(K, \kappa) \in \Psi$, with an equilibrium route flow pattern $\left(\mathbf{f}^{\mathrm{HDV}}, \mathbf{f}^{\mathrm{CAV}}\right.$, $\left.\mathbf{d}^{\mathrm{HDV}}, \mathbf{d}^{\mathrm{CAV}}\right)$, the equilibrium credit price $p^{*}$ is unique if the same condition in Proposition 3 is satisfied.

Proof. Note that the ratio of $f_{r_{1}, w}^{m^{*}}$ and $f_{r_{2}, w}^{m^{*}}$ is equal to the middle term of equation (19). The only difference is the different VOT and extra expense $c_{0}$ included in the travel cost. Following the same fashion in the proof of Proposition 3 , we can get

$$
p^{*}=\frac{\left(\ln f_{r_{1}, w}^{m^{*}}-\ln f_{r_{2}, w}^{m^{*}}\right)+\theta_{m} \beta_{m}\left(t_{r_{1}, w}^{*}-t_{r_{2}, w}^{*}\right)}{\theta_{m}\left(\kappa_{r_{2}, w}-\kappa_{r_{1}, w}\right)} .
$$

The credit price at market equilibrium is uniquely determined. It should be noted that the extra expense of CAV has no direct relationship with equilibrium credit price.

3.3. Solution Algorithm. With some modifications, the solution algorithm proposed in Section 2.3 can be also applied to the case with endogenous CAV penetration rate, since the RSRS algorithm is proved to be competent in solving nested-logit problem [28]. The modified version is given as follows.

Step 0. Determine the constant of tolerances $G_{0}$ and $\varepsilon_{p}$ and the sequence of move sizes $\alpha_{n}$ given by (22). Randomly initialize the credit price $p_{i}>0$. Set $i:=1$.

Step 1. Uniformly distribute the O-D demands for $\mathrm{HDV}$ and CAV for each O-D pair, denoted by $\mathbf{d}_{j}^{\mathrm{HDV}}$ and $\mathbf{d}_{j}^{\mathrm{CAV}}$ in vectors (initialize $p_{\mathrm{CAV}}$ as $50 \%$ ). Set $j:=1$.
Step 2. Update the travel demand for HDV and CAV.

(i) Run an RSRS algorithm with $p_{i}, \mathbf{d}_{j}^{\mathrm{HDV}}$, and $\mathbf{d}_{j}^{\mathrm{CAV}}$ and acquire the flow patterns $\mathbf{f}_{j}^{\mathrm{HDV}}$ and $\mathbf{f}_{j}^{\mathrm{CAV}}$.

(ii) Update the expected minimum perceived travel $\operatorname{cost} \bar{c}_{w}^{m}$ by (A.12).

(iii) Given the expected minimum perceived travel cost, update travel demands $\mathbf{d}_{j+1}^{\mathrm{HDV}}$ and $\mathbf{d}_{j+1}^{\mathrm{CAV}}$ according to equation (B.10).

(iv) If the convergence criterion,

$$
\sum_{m \in M} \frac{\sqrt{\sum_{w \in W}\left(d_{w}^{m}(j+1)-d_{w}^{m}(j)\right)^{2}}}{\sum_{w \in W} d_{w}^{m}(j)} \leq \varepsilon_{d},
$$

is satisfied, calculate $\mathbf{f}_{j+1}^{\mathrm{HDV}}$ and $\mathbf{f}_{j+1}^{\mathrm{CAV}}$ and return $\mathbf{f}^{\mathrm{HDV}}=\mathbf{f}_{j+1}^{\mathrm{HDV}}$, $\mathbf{f}^{\mathrm{CAV}}=\mathbf{f}_{j+1}^{\mathrm{CAV}}, \mathbf{d}^{\mathrm{HDV}}=\mathbf{d}_{j+1}^{\mathrm{HDV}}$, and $\mathbf{d}^{\mathrm{CAV}}=\mathbf{d}_{j+1}^{\mathrm{CAV}}$. Otherwise, set $j:=j+1$ and go to (i).

Step 3. Check the scheme validity. If $\sum_{a \in A} \kappa_{a} v_{a}$ $\left(\mathbf{f}^{\mathrm{HDV}}, \mathbf{f}^{\mathrm{CAV}}\right) \leq K$, set $p_{i+1}=0$. Otherwise, set the credit price $p_{i+1}$ with $p_{i+1}=\max \left(0, p_{i}+(1 / i)\left(\sum_{a \in A} \kappa_{a} v_{a}\right.\right.$ $\left.\left.\left(\mathbf{f}^{\mathrm{HDV}}, \mathbf{f}^{\mathrm{CAV}}\right)-K\right)\right)$.

Step 4. If the convergence criterion (25) is satisfied, stop the algorithm and return $p^{*}=p_{i+1}$ and $\left(\mathbf{f}^{\mathrm{HDV}}, \mathbf{f}^{\mathrm{CAV}}\right.$, $\left.\mathbf{d}^{\mathrm{HDV}}, \mathbf{d}^{\mathrm{CAV}}\right)$. Otherwise, set $i:=i+1$ and go to Step 1 .

As we can see, a middle iteration is added to the iterative process to get the optimal travel demands for HDVs and CAVs. The vehicle type-specific travel demand is updated based on the optimal path flow pattern acquired from the inner iteration (RSRS algorithm) till the successive results are sufficiently close. The procedure will also be terminated after the difference between two successive credit prices is within a tolerance.

3.4. Numerical Analysis. In this section, we apply the above algorithm to the Nguyen and Dupuis network [63] to study the traffic equilibrium with endogenous CAV penetration under TCS. As shown in Figure 6, the network consists of 13 nodes, 19 links, 4 OD pairs, and 25 routes. For O-D pairs (1, $2),(1,3),(4,2)$, and $(4,3)$, the travel demands are 400,800 , 600 , and 200 units, respectively. The dispersion parameters and parameters for RSRS algorithm are consistent with Section 2. The values of time for HDV and CAV users are the same as those in Section 2. We assume that the total amount of distributed credits is 18000 , and each traveler on the network is initially distributed 9 credits. The detailed information of the network and credit scheme is given in Table 2.

The convergence results for unit credit price are shown in Figure 7. It can be found that the it takes about 50 iterations to achieve the objective values, seemingly faster than the case with exogenous CAV penetration rate. Nonetheless, we cannot conclude that it converges faster for larger network or something because three types of iterations are involved in the algorithm given in Section 3.3. It is also meaningless to compare the convergence rate for solving traffic equilibrium with exogenous and endogenous CAV penetration rate. 


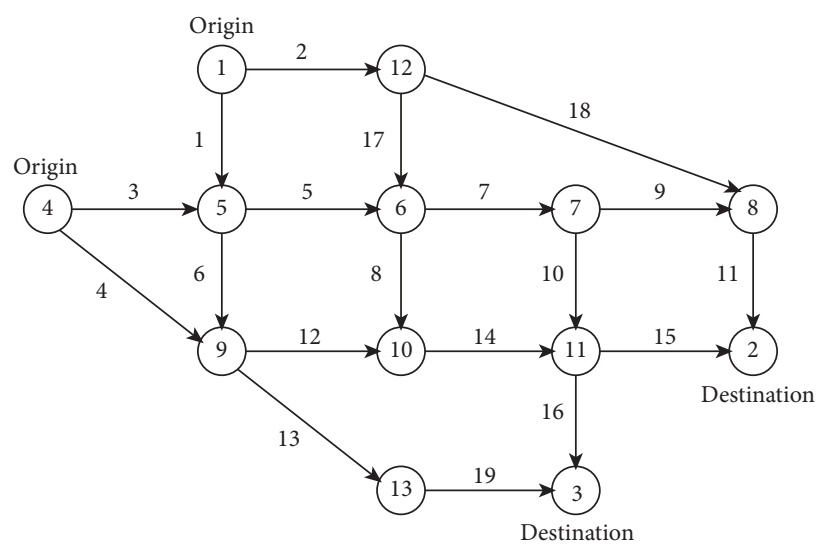

Figure 6: The Nguyen and Dupuis network.

TABle 2: Input data of Nguyen and Dupuis network.

\begin{tabular}{|c|c|c|c|c|}
\hline Link & Free flow travel time & Capacity for HDV & Capacity for CAV & Credit charge \\
\hline 1 & 7 & 300 & 600 & 2 \\
\hline 2 & 9 & 200 & 400 & 2 \\
\hline 3 & 9 & 200 & 400 & 2 \\
\hline 4 & 12 & 200 & 400 & 2 \\
\hline 5 & 3 & 350 & 700 & 2 \\
\hline 6 & 9 & 400 & 800 & 2 \\
\hline 7 & 5 & 500 & 1000 & 2 \\
\hline 8 & 13 & 250 & 500 & 3 \\
\hline 9 & 5 & 250 & 500 & 2 \\
\hline 10 & 9 & 300 & 600 & 3 \\
\hline 11 & 9 & 500 & 1000 & 2 \\
\hline 12 & 10 & 550 & 1100 & 2 \\
\hline 13 & 9 & 200 & 400 & 2 \\
\hline 14 & 6 & 400 & 800 & 2 \\
\hline 15 & 9 & 300 & 600 & 2 \\
\hline 16 & 8 & 300 & 600 & 2 \\
\hline 17 & 7 & 200 & 400 & 2 \\
\hline 18 & 14 & 300 & 600 & 2 \\
\hline 19 & 11 & 200 & 400 & 2 \\
\hline
\end{tabular}

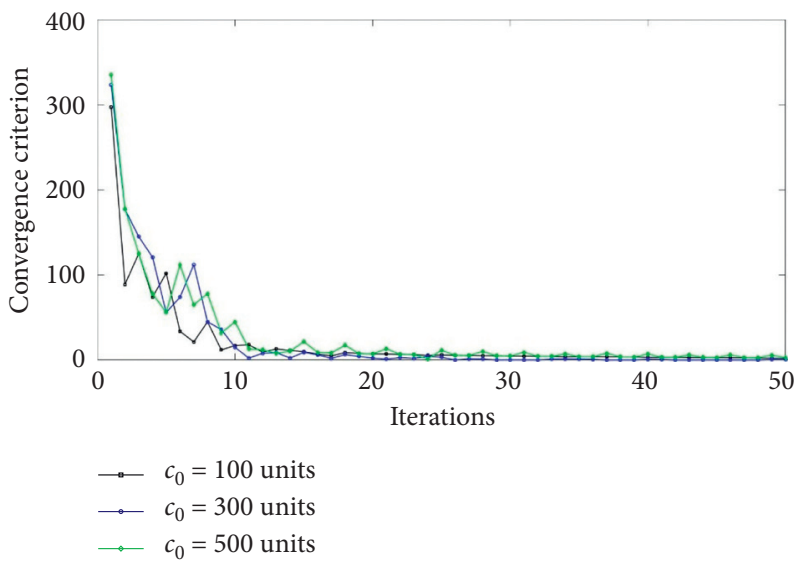

FIGURE 7: Convergence results for unit credit price with various extra expenses. 


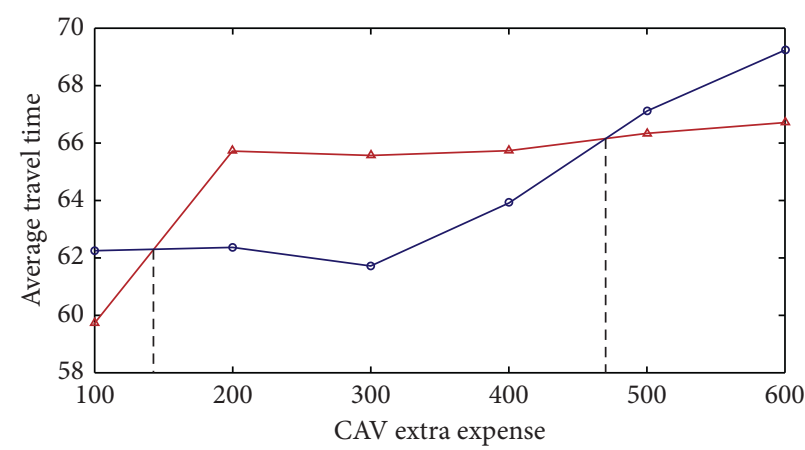

$\rightarrow$ HDV users
$\rightarrow$ CAV users

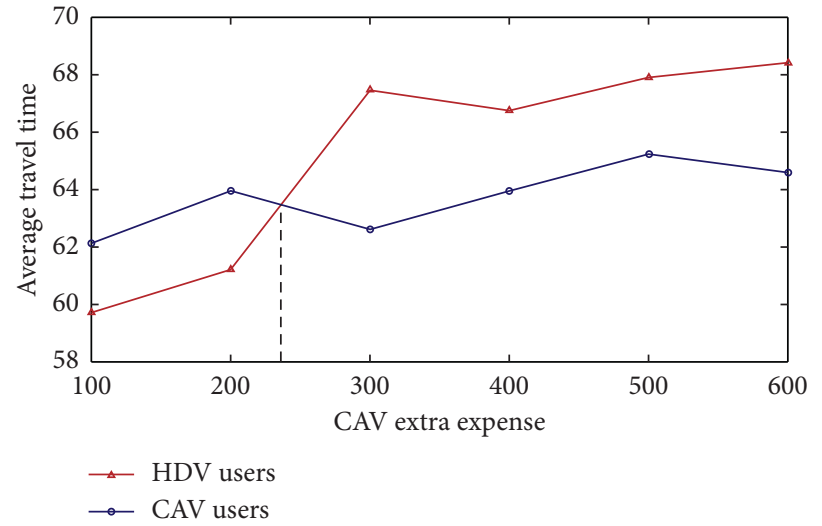

(b)

(a)

FIgUre 8: Average travel time for HDV and CAV users. (a) Average travel time without AVCC link. (b) Average travel time with AVCC link.

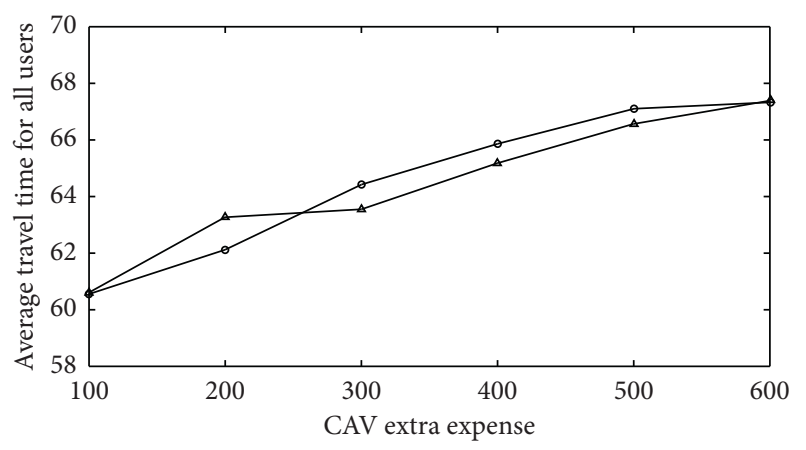

$\rightarrow$ Without AVCC link

$\therefore$ With AVCC link

Figure 9: Average travel time for all network users.

Similarly, we convert link 8 and link 10 to AVCC links to investigate the impacts of the deployment of AVCC links.

3.4.1. Main Findings. Figure 8 shows the average travel time for HDV and CAV users. From Figure 8(a), we can see that the HDV user experiences higher travel time within an approximate range of $140 \leq c_{0} \leq 470$ units. When the extra expense is small, more travelers will choose to travel by CAVs. With a lower VOT, they weigh less on travel time than the credit cost and thus lead to a higher average travel time than HDVs. A higher average travel time of CAV is also found when the extra expense goes beyond the upper bound. The gap will become larger as the expense increases, since the CAV's average travel time keeps increasing while the HDV's tends to be stable according to the figure. This implies that either a lower or higher expense may suppress the advantages of CAVs, resulting in an actual higher travel time in the network.

By contrast, the deployment of AVCC links can avoid the average travel time of CAV from increasing with its extra expenses. From Figure 8(b), we can see that when the extra expense is higher than about 230 units, the CAV user experiences lower average travel time than HDV user.

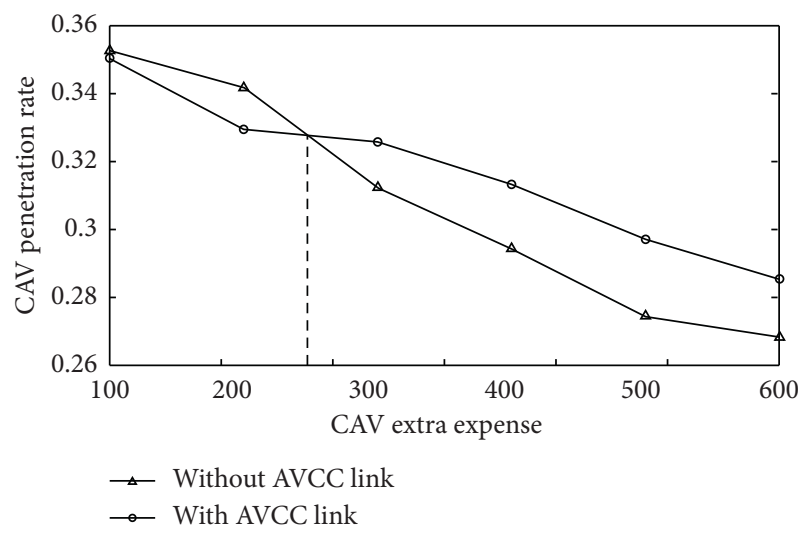

Figure 10: CAV penetration rate under various extra expenses.

It can be observed from Figure 9 that the average travel time for all users in presence of AVCC links is lower when $c_{0}$ is below 260, which indicates that a higher expense on CAV can also worsen the improvement brought by AVCC links on all users.

To figure out the reason for the improvement brought by AVCC links, we then look at the impact of CAV extra expense on the CAV penetration rate for the network. Figure 10 shows the variations in CAV penetration rate before and after the deployment of AVCC links. It can be seen that the deployment of AVCC links can slow down the decline rate of CAV penetration with increasing expense. This is because the conversion of regular links to AVCC links decreases the travel cost of CAVs and thus increases the utility for travelers to travel by CAVs.

Also, the impact of CAV expense on credit market is investigated as shown in Figure 11. It can be seen that the credit price does not change too much as the extra expense increases. Similarly, the deployment of AVCC link can lower the credit price. From Figure 11(b), we can see that the deployment of AVCC links can also stimulate credit trading, but the stimulating effect decreases as CAV expense increases. 


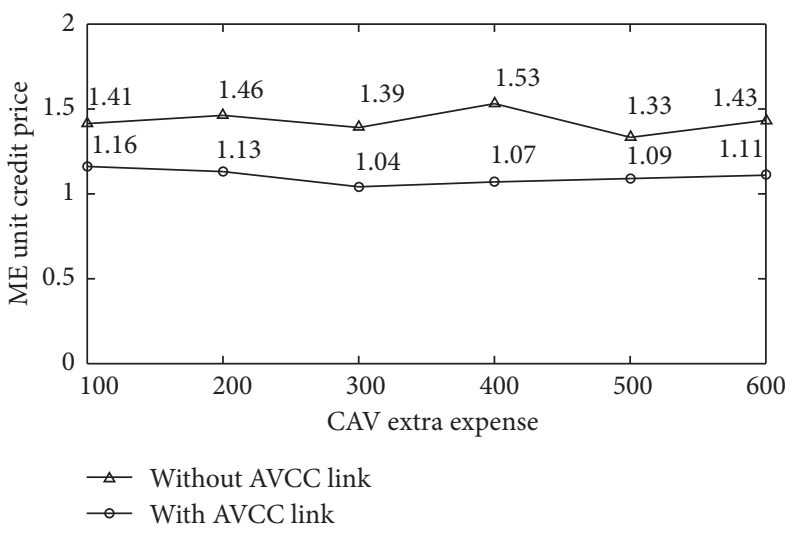

(a)

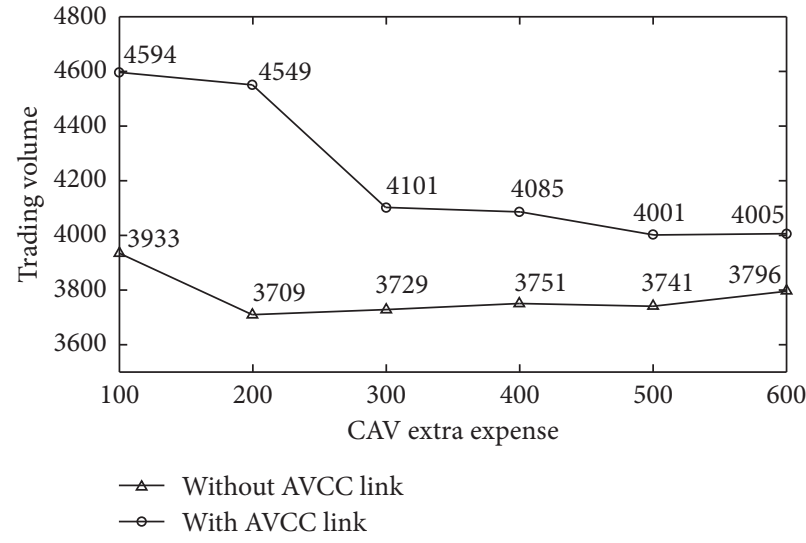

(b)

Figure 11: States of credit market under various CAV extra expenses. (a) Market equilibrium unit credit price. (b) Trading volume among travelers.

\section{Concluding Remarks}

In this paper, the traffic equilibriums for mixed traffic flows of HDV and CAV under tradable credit scheme are established and formulated as two VI problems with exogenous and endogenous CAV penetration rate, respectively. A modified Lagrangian dual method embedded with a revised Smith's route-swapping algorithm is proposed to solve the problems.

Based on the results from example networks, the impacts of CAV penetration and its extra expense on network performance are investigated. The potentials of AVCC links to improve the network performance are demonstrated. The main findings with exogenous and endogenous penetration rate are given as follows.

With exogenous CAV penetration rate,

(i) The deployment of AVCC links can improve the efficiency of TCS due to its better guidance for the HDVs with high perception variances to choose the routes with lower actual travel cost;

(ii) The market equilibrium credit price can be reduced by the penetration of CAV and further lowered by the deployment of AVCC link. Meanwhile, the deployment of AVCC link can stimulate credit trading among travelers and increase the trading volume in the credit market.

With endogenous CAV penetration rate,

(i) The CAV extra expense can decline the CAV penetration in the network. A high extra expense of
CAV may suppress the advantages of CAVs, resulting in a worse performance than HDVs in the network. Promisingly, the deployment of AVCC links can alleviate the negative impact of high expense of CAV by slowing down the decline rate of $\mathrm{CAV}$ penetration resulting from increasing extra expense.

(ii) The impact of CAV expense on the credit price is very limited. But it can suppress credit-trading in the credit market. Still, the deployment of AVCC links can stimulate credit trading and reduce credit price.

We believe this work can provide an insight for CAV management under tradable credit scheme when CAVs are commonly available for people. Further researches can be carried out in the following two aspects. On the one hand, a more sophisticated model such as cross-nested-logit model can be applied to describe the travelers' behavior more accurately, since it can overcome the overlap issue in logitbased model. On the other hand, the deployment of AVCC links can be formulated as a network design problem, which can be solved to find the optimal strategy of deployment under a given TCS.

\section{Appendix}

\section{A. Proof of Proposition 1}

The Lagrangian function of VI problem (16) is

$$
L=\sum_{w \in W} \sum_{r \in R^{w}} \sum_{m \in M}\left(c_{r, w}^{m^{*}}+\frac{1}{\theta_{m}} \ln f_{r, w}^{m^{*}}\right) f_{r, w}^{m}+p\left(\sum_{a \in A} \kappa_{a} v_{a}^{*}-K\right)+\sum_{w \in W} \sum_{m \in M} \mu_{w}^{m}\left(d_{w}^{m}-\sum_{r \in R^{w}} f_{r, w}^{m}\right) .
$$

The Karush-Kuhn-Tucker (KKT) conditions for this problem are 


$$
f_{r, w}^{m^{*}}\left(c_{r, w}^{m^{*}}+\frac{1}{\theta_{m}} \ln f_{r, w}^{m^{*}}-\mu_{w}^{m}\right)=0, \quad r \in R^{w}, w \in W, m \in M,
$$

$$
c_{r, w}^{m^{*}}+\frac{1}{\theta_{m}} \ln f_{r, w}^{m^{*}}-\mu_{w}^{m} \geq 0, \quad r \in R^{w}, w \in W, m \in M,
$$

$$
\begin{aligned}
f_{r, w}^{m^{*}} & \geq 0, \quad r \in R^{w}, w \in W, m \in M, \\
\sum_{r \in R^{w}} f_{r, w}^{m^{*}} & =d_{w}^{m}, \quad w \in W, m \in M,
\end{aligned}
$$

$$
\begin{aligned}
\left(\sum_{a \in A} \kappa_{a} v_{a}^{*}-K\right) p^{*} & =0, \\
\sum_{a \in A} \kappa_{a} v_{a}^{*}-K & \leq 0, \quad p^{*} \geq 0 .
\end{aligned}
$$

From equation (A.2), for $f_{r, w}^{m^{*}} \geq 0$, we have

$$
c_{r, w}^{m^{*}}+\frac{1}{\theta_{m}} \ln f_{r, w}^{m^{*}}-\mu_{w}^{m}=0 .
$$

It can be transferred to

$$
f_{r, w}^{m^{*}}=\exp \left(-\theta_{m} c_{r, w}^{m^{*}}\right) \cdot \exp \left(\theta_{m} \mu_{w}^{m}\right) .
$$

Summing equation (A.9) for all paths for the same O-D pair $w$ and considering constraint (5), we have

$$
d_{w}^{m}=\exp \left(\theta_{m} \mu_{w}^{m}\right) \cdot \sum_{r \in R^{w}} \exp \left(-\theta_{m} c_{r, w}^{m}\right)
$$

Then, $\mu_{w}^{m}$ can be calculated from equation (A.10), i.e.,

$$
\mu_{w}^{m}=\frac{1}{\theta_{m}} \ln d_{w}^{m}+\bar{c}_{w}^{m}
$$

where

$$
\bar{c}_{w}^{m}=-\frac{1}{\theta_{m}} \ln \sum_{r \in R^{w}} \exp \left(-\theta_{m} c_{r, w}^{m}\right)
$$

is the expected minimum perceived travel cost of vehicle type $m$ on O-D pair $w$. Substituting equation (A.11) into equation (A.8), we have

$$
f_{r, w}^{m^{*}}=\frac{d_{w}^{m} \cdot \exp \left(-\theta_{m} c_{r, w}^{m^{*}}\right)}{\sum_{k \in R^{w}} \exp \left(-\theta_{m} c_{k, w}^{m^{*}}\right)}, \quad r \in R^{w}, w \in W, m \in M .
$$

The above equation is equivalent to the SUE conditions (12) and (13). Besides, equations (A.6) and (A.7) are equivalent to the credit market equilibrium conditions (14) and (15). Equation (A.4) represents the nonnegative route flows and equation (A.5) represents the flow conservation. The Lagrange multipliers $p$ and $\mu_{w}^{m}, w \in W, m \in M$ correspond to the unit credit price at market equilibrium and the minimal generalized travel cost for O-D pair $w$ for vehicle type $m$ at user equilibrium. This completes the proof.

\section{B. Proof of Proposition 4}

The Lagrangian function of VI problem (33) is

$$
\begin{aligned}
L= & \sum_{w \in W} \sum_{r \in R^{w}} \sum_{m \in M}\left(c_{r, w}^{m^{*}}+\frac{1}{\theta_{m}} \ln f_{r, w}^{m^{*}}\right) f_{r, w}^{m}+\sum_{w \in W} \sum_{m \in M}\left(1-\frac{1}{\theta_{m}}\right) \ln d_{w}^{m^{*}} d_{w}^{m}+p\left(\sum_{a \in A} \kappa_{a} v_{a}^{*}-K\right) \\
& +\sum_{w \in W} \sum_{m \in M} \mu_{w}^{m}\left(d_{w}^{m}-\sum_{r \in R^{w}} f_{r, w}^{m}\right)+\sum_{w \in W} \lambda_{w}\left(d_{w}-\sum_{m \in M} d_{w}^{m}\right) .
\end{aligned}
$$

The Karush-Kuhn-Tucker (KKT) conditions for this problem are as follows.

Consider equations (A.2)-(A.7) and

$$
d_{w}^{m^{*}}\left(\left(1-\frac{1}{\theta_{m}}\right) \ln d_{w}^{m^{*}}+\mu_{w}^{m}-\lambda_{w}\right)=0, \quad w \in W, m \in M
$$

$$
\left(1-\frac{1}{\theta_{m}}\right) \ln d_{w}^{m^{*}}+\mu_{w}^{m}-\lambda_{w} \geq 0, \quad w \in W, m \in M,
$$

$$
d_{w}^{m^{*}} \geq 0, \quad w \in W, m \in M,
$$

$$
\sum_{m \in M} d_{w}^{m^{*}}=d_{w}, \quad w \in W
$$

From Equation (B.2), for $d_{w}^{m^{*}} \geq 0$, we have

$$
\left(1-\frac{1}{\theta_{m}}\right) \ln d_{w}^{m^{*}}+\mu_{w}^{m}-\lambda_{w}=0
$$

With Equation (A.12), it can be transferred to

$$
d_{w}^{m^{*}}=\exp \left(\lambda_{w}\right) \cdot \exp \left(-\bar{c}_{w}^{m}\right) .
$$

Summing equation (B.7) for HDVs and CAVs for the same O-D pair $w$ and considering constraint (6), we have

$$
d_{w}=\exp \left(\lambda_{w}\right) \cdot \sum_{m \in M} \exp \left(-\bar{c}_{w}^{m}\right) .
$$


Then, $\lambda_{w}$ can be calculated from equation (B.8), i.e.,

$$
\lambda_{w}=\ln d_{w}-\ln \sum_{m \in M} \exp \left(-\bar{c}_{w}^{m}\right)
$$

Substituting equation (B.9) into equation (B.7), we have

$$
d_{w}^{m^{*}}=\frac{d_{w} \cdot \exp \left(-\bar{c}_{w}^{m}\right)}{\sum_{m^{\prime} \in M} \exp \left(-\bar{c}_{w}^{m^{\prime}}\right)}, \quad w \in W, m \in M .
$$

Together with equation (A.12), we have

$$
\begin{array}{r}
f_{r, w}^{m^{*}}=\frac{\exp \left(-\theta_{m} c_{r, w}^{m}\right)}{\sum_{k \in R^{w}} \exp \left(-\theta_{m} c_{k, w}^{m}\right)} \cdot \frac{\exp \left(-\bar{c}_{w}^{m}\right)}{\sum_{m^{\prime} \in M} \exp \left(-\bar{c}_{w}^{m^{\prime}}\right)} \cdot d_{w}, \\
\quad r \in R^{w}, w \in W, m \in M .
\end{array}
$$

The above equations are equivalent to the SUE conditions (31) and (32). Apart from equivalence demonstrated in Proposition 1, equation (B.4) represents the nonnegative travel demand and equation (B.5) represents the demand conservation. The Lagrange multipliers $\lambda_{w}, w \in W$ correspond to the minimal generalized utility for traveling on $\mathrm{O}-\mathrm{D}$ pair $w$ by either HDV or CAV at user equilibrium. This completes the proof.

\section{Data Availability}

No data were used during the study.

\section{Conflicts of Interest}

The authors declare that there are no conflicts of interest regarding the publication of this paper.

\section{References}

[1] Google and Waymo, "Google self-driving car project,"Waymo, Mountain View, CA, USA, 2016, URL https://www. google.com/selfdrivingcar/.

[2] Baidu, "Autonomous driving unit,"Baidu, USA, 2018, URL http://usa.baidu.com/adu/.

[3] nuTonomy, "nuTonomy and Lyft launch boston self-driving pilot," nuTonomy, Cambridge, MA, USA, 2019, https://www. nutonomy.com/company-news/nutonomy-and-lyft-launchboston-self-drivingpilot/.

[4] A. Miller, "Some of the companies that are working on driverless car technology," ABC News, Newyork, NY, USA, 2019, https://abcnews.go.com/US/companies-workingdriverless-car-technology/\%0Astory?id=53872985.

[5] NTC, "Changing driving laws to support automated vehicles," 2017, http://www.ntc.gov.au/Media/Reports/(E5695ACE993C-618F-46E1-A876391B8CD9).pdf.

[6] M. Schaub and A. Zhao, "China: put self-driving cars into gear," King \& Wood Mallesons, Hong kong, 2018, https:// www.chinalawinsight.com/2017/06/articles/corporate/chinaput-self-driving-cars-intogear/\#\%0Amore-11459.

[7] S. Slone, "State laws on autonomous vehicles," 2016, http:// knowledgecenter.csg.org/kc/content/state-laws-autonomousvehicles.

[8] S. E. Shladover, D. Su, and X.-Y. Lu, "Impacts of cooperative adaptive cruise control on freeway traffic flow,"
Transportation Research Record: Journal of the Transportation Research Board, vol. 2324, no. 1, pp. 63-70, 2012.

[9] V. Milanes, S. E. Shladover, J. Spring, C. Nowakowski, H. Kawazoe, and M. Nakamura, "Cooperative adaptive cruise control in real traffic situations," IEEE Transactions on Intelligent Transportation Systems, vol. 15, no. 1, pp. 296-305, 2014.

[10] M. W. Levin and S. D. Boyles, "A multiclass cell transmission model for shared human and autonomous vehicle roads," Transportation Research Part C: Emerging Technologies, vol. 62, pp. 103-116, 2016.

[11] L. Ye and T. Yamamoto, "Evaluating the impact of connected and autonomous vehicles on traffic safety," Physica A: Statistical Mechanics and its Applications, vol. 526, Article ID 121009, 2019.

[12] A. Papadoulis, M. Quddus, and M. Imprialou, "Evaluating the safety impact of connected and autonomous vehicles on motorways," Accident Analysis \& Prevention, vol. 124, pp. 12-22, 2019.

[13] J. B. Greenblatt and S. Saxena, "Autonomous taxis could greatly reduce greenhouse-gas emissions of US light-duty vehicles," Nature Climate Change, vol. 5, no. 9, pp. 860-863, 2015.

[14] A. C. Mersky and C. Samaras, "Fuel economy testing of autonomous vehicles," Transportation Research Part C: Emerging Technologies, vol. 65, pp. 31-48, 2016.

[15] D. J. Fagnant and K. Kockelman, "Preparing a nation for autonomous vehicles: opportunities, barriers and policy recommendations," Transportation Research Part A: Policy and Practice, vol. 77, pp. 167-181, 2015.

[16] S. Zong, "How connected autonomous vehicles would affect our world? - a literature review on the impacts of CAV on road capacity, environment and public attitude," MATEC Web Conf, vol. 296, Article ID 01007, 2019.

[17] H. K. Lo and W. Y. Szeto, "Modeling advanced traveler information services: static versus dynamic paradigms," Transportation Research Part B: Methodological, vol. 38, no. 6, pp. 495-515, 2004.

[18] H. K. Lo and W. Y. Szeto, "A methodology for sustainable traveler information services," Transportation Research Part B: Methodological, vol. 36, no. 2, pp. 113-130, 2002.

[19] H. Tsuji, R. Takahashi, H. Kawashima, and Y. Yamamoto, "A stochastic approach for estimating the effectiveness of a route guidance system and its related parameters," Transportation Science, vol. 19, no. 4, pp. 333-351, 1985.

[20] H. Yang, "Multiple equilibrium behaviors and advanced traveler information systems with endogenous market penetration," Transportation Research Part B: Methodological, vol. 32, no. 3, pp. 205-218, 1998.

[21] H. Yang, R. Kitamura, P. P. Jovanis, K. M. Vaughn, and M. A. Abdel-Aty, "Exploration of route choice behavior with advanced traveler information using neural network concepts,” Transportation, vol. 20, no. 2, pp. 199-223, 1993.

[22] H. Yang and Q. Meng, "Modeling user adoption of advanced traveler information systems: dynamic evolution and stationary equilibrium," Transportation Research Part A: Policy and Practice, vol. 35, no. 10, pp. 895-912, 2001.

[23] Y. Yin and H. Yang, "Simultaneous determination of the equilibrium market penetration and compliance rate of advanced traveler information systems," Transportation Research Part A: Policy and Practice, vol. 37, no. 2, pp. 165-181, 2003.

[24] Z. Chen, F. He, L. Zhang, and Y. Yin, “Optimal deployment of autonomous vehicle lanes with endogenous market 
penetration," Transportation Research Part C: Emerging Technologies, vol. 72, pp. 143-156, 2016.

[25] Z. Chen, F. He, Y. Yin, and Y. Du, "Optimal design of autonomous vehicle zones in transportation networks," Transportation Research Part B: Methodological, vol. 99, pp. 44-61, 2017.

[26] N. Jiang, "Optimal signal design for mixed equilibrium networks with autonomous and regular vehicles," Journal of Advanced Transportation, vol. 2017, Article ID 5649823, 13 pages, 2017.

[27] K. Zhang and Y. Nie, "Mitigating the impact of selfish routing: an optimal-ratio control scheme (ORCS) inspired by autonomous driving," Transportation Research Part C: Emerging Technologies, vol. 87, pp. 75-90, 2018.

[28] J. Wang, S. Peeta, and X. He, "Multiclass traffic assignment model for mixed traffic flow of human-driven vehicles and connected and autonomous vehicles," Transportation Research Part B: Methodological, vol. 126, pp. 139-168, 2019.

[29] H.-J. Huang and Z.-C. Li, "A multiclass, multicriteria logitbased traffic equilibrium assignment model under ATIS," European Journal of Operational Research, vol. 176, no. 3, pp. 1464-1477, 2007.

[30] D. Wu, Y. Yin, S. Lawphongpanich, and H. Yang, "Design of more equitable congestion pricing and tradable credit schemes for multimodal transportation networks," Transportation Research Part B: Methodological, vol. 46, no. 9, pp. 1273-1287, 2012.

[31] A. Perrels, "User response and equity considerations regarding emission cap-and-trade schemes for travel," Energy Efficiency, vol. 3, no. 2, pp. 149-165, 2010.

[32] H. Yang and X. Wang, "Managing network mobility with tradable credits," Transportation Research Part B: Methodological, vol. 45, no. 3, pp. 580-594, 2011.

[33] Y. Bao, Z. Gao, M. Xu, and H. Yang, "Tradable credit scheme for mobility management considering travelers' loss aversion," Transportation Research Part E: Logistics and Transportation Review, vol. 68, pp. 138-154, 2014.

[34] S. Grant-Muller and M. Xu, "The role of tradable credit schemes in road traffic congestion management," Transport Reviews, vol. 34, no. 2, 2014.

[35] N. Shirmohammadi, M. Zangui, Y. Yin, and Y. Nie, "Analysis and design of tradable credit schemes under uncertainty," Transportation Research Record: Journal of the Transportation Research Board, vol. 2333, no. 1, pp. 27-36, 2013.

[36] L.-J. Tian, H. Yang, and H.-J. Huang, “Tradable credit schemes for managing bottleneck congestion and modal split with heterogeneous users," Transportation Research Part E: Logistics and Transportation Review, vol. 54, pp. 1-13, 2013.

[37] X. Wang, H. Yang, D. Zhu, and C. Li, "Tradable travel credits for congestion management with heterogeneous users," Transportation Research Part E: Logistics and Transportation Review, vol. 48, no. 2, pp. 426-437, 2012.

[38] F. Xiao, Z. Qian, and H. M. Zhang, "Managing bottleneck congestion with tradable credits," Transportation Research Part B: Methodological, vol. 56, pp. 1-14, 2013.

[39] D.-L. Zhu, H. Yang, C.-M. Li, and X.-L. Wang, "Properties of the multiclass traffic network equilibria under a tradable credit scheme," Transportation Science, vol. 49, no. 3, pp. 519-534, 2015.

[40] N. Shirmohammadi and Y. Yin, "Tradable credit scheme to control bottleneck queue length," Transportation Research Record: Journal of the Transportation Research Board, vol. 2561, no. 1, pp. 53-63, 2016.
[41] M. Miralinaghi and S. Peeta, "Multi-period equilibrium modeling planning framework for tradable credit schemes," Transportation Research Part E: Logistics and Transportation Review, vol. 93, pp. 177-198, 2016.

[42] Y. Bao, Z. Gao, H. Yang, M. Xu, and G. Wang, "Private financing and mobility management of road network with tradable credits," Transportation Research Part A: Policy and Practice, vol. 97, pp. 158-176, 2017.

[43] M. Miralinaghi and S. Peeta, "A multi-period tradable credit scheme incorporating interest rate and traveler value-of-time heterogeneity to manage traffic system emissions," Frontiers in Built Environment, vol. 4, 2018.

[44] M. Miralinaghi and S. Peeta, "Promoting zero-emissions vehicles using robust multi-period tradable credit scheme," Transportation Research Part D: Transport and Environment, vol. 75, pp. 265-285, 2019.

[45] R. Y. Guo, H. J. Huang, and H. Yang, "Tradable credit scheme for control of evolutionary traffic flows to system optimum: model and its convergence," Networks and Spatial Economics, vol. 19, no. 3, pp. 833-868, 2019.

[46] G. Wang, M. Xu, S. Grant-Muller, and Z. Gao, "Combination of tradable credit scheme and link capacity improvement to balance economic growth and environmental management in sustainable-oriented transport development: a bi-objective bilevel programming approach," Transportation Research Part A: Policy and Practice, 2018, in Press.

[47] M. Miralinaghi, S. Peeta, X. He, and S. V. Ukkusuri, "Managing morning commute congestion with a tradable credit scheme under commuter heterogeneity and market loss aversion behavior," Transportmetrica B: Transport Dynamics, vol. 7, no. 1, pp. 1780-1808, 2019.

[48] N. Dogterom, Y. Bao, M. Xu, and D. Ettema, "Acceptability of a tradable driving credit scheme in the Netherlands and Beijing," Case Studies on Transport Policy, vol. 6, no. 4, pp. 499-509, 2018.

[49] N. Dogterom, D. Ettema, and M. Dijst, "Activity-travel adaptations in response to a tradable driving credits scheme," Transport Policy, vol. 72, pp. 79-88, 2018.

[50] Y. Tian, Y.-C. Chiu, and J. Sun, "Understanding behavioral effects of tradable mobility credit scheme: an experimental economics approach," Transport Policy, vol. 81, pp. 1-11, 2019.

[51] M. Xu and S. Grant-Muller, "Trip mode and travel pattern impacts of a tradable credits scheme: a case study of Beijing," Transport Policy, vol. 47, pp. 72-83, 2016.

[52] F. Han and L. Cheng, "Stochastic user equilibrium model and its algorithm considering tradable credit scheme," Dongnan Daxue Xuebao (Ziran Kexue Ban)/Journal of Southeast University (Natural Science Edition), vol. 46, no. 1, pp. 215-220, 2016.

[53] F. Han and L. Cheng, "Stochastic user equilibrium model with a tradable credit scheme and application in maximizing network reserve capacity," Engineering Optimization, vol. 49, no. 4, pp. 549-564, 2017.

[54] Z. Liu and Z. Song, "Strategic planning of dedicated autonomous vehicle lanes and autonomous vehicle/toll lanes in transportation networks," Transportation Research Part C: Emerging Technologies, vol. 106, pp. 381-403, 2019.

[55] N. Mehr and R. Horowitz, "How will the presence of autonomous vehicles affect the equilibrium state of traffic networks?" IEEE Transactions on Control of Network Systems, vol. 20, no. 1, 2020.

[56] M. Noruzoliaee, B. Zou, and Y. Liu, "Roads in transition: integrated modeling of a manufacturer-traveler-infrastructure system in a mixed autonomous/human driving 
environment," Transportation Research Part C: Emerging Technologies, vol. 90, pp. 307-333, 2018.

[57] V. A. C. van den Berg and E. T. Verhoef, "Autonomous cars and dynamic bottleneck congestion: the effects on capacity, value of time and preference heterogeneity," Transportation Research Part B: Methodological, vol. 94, pp. 43-60, 2016.

[58] F. Facchinei and J. S. Pang, "Finite-dimensional variational inequalities and complementarity problems," Finite-Dimensional Variational Inequalities and Complementarity Problems, Springer, Newyork, NY, USA, 2003.

[59] R.-Y. Guo, H. Yang, and H.-J. Huang, "A discrete rational adjustment process of link flows in traffic networks," Transportation Research Part C: Emerging Technologies, vol. 34, pp. 121-137, 2013.

[60] M. J. Smith and D. P. Watling, "A route-swapping dynamical system and Lyapunov function for stochastic user equilibrium," Transportation Research Part B: Methodological, vol. 85, pp. 132-141, 2016.

[61] H.-J. Huang and W. H. K. Lam, "Modeling and solving the dynamic user equilibrium route and departure time choice problem in network with queues," Transportation Research Part B: Methodological, vol. 36, no. 3, pp. 253-273, 2002.

[62] S. Peeta and T.-H. Yang, "Stability issues for dynamic traffic assignment," Automatica, vol. 39, no. 1, pp. 21-34, 2003.

[63] S. Nguyen and C. Dupuis, "An efficient method for computing traffic equilibria in networks with asymmetric transportation costs," Transportation Science, vol. 18, no. 2, pp. 185-202, 1984.

[64] T. Miwa, Y. Okada, and T. Morikawa, “Applying a structured dispersion parameter to multiclass stochastic user equilibrium assignment model," Transportation Research Record: Journal of the Transportation Research Board, vol. 2196, no. 1, pp. 142-149, 2010. 